\title{
Approximation-Based Fixed-Time Adaptive Tracking Control for a Class of Uncertain Nonlinear Pure-Feedback Systems
}

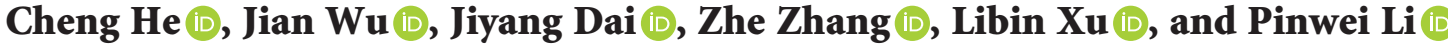 \\ ${ }^{1}$ School of Information Engineering, Nanchang Hangkong University, Nanchang 330063, China \\ Correspondence should be addressed to Jian Wu; 78313993@qq.com
}

Received 23 November 2019; Revised 1 March 2020; Accepted 28 March 2020; Published 27 April 2020

Academic Editor: Matilde Santos

Copyright (c) 2020 Cheng He et al. This is an open access article distributed under the Creative Commons Attribution License, which permits unrestricted use, distribution, and reproduction in any medium, provided the original work is properly cited.

\begin{abstract}
This paper examines approximation-based fixed-time adaptive tracking control for a class of uncertain nonlinear pure-feedback systems. Novel virtual and actual controllers are designed that resolve the meaninglessness of virtual and actual controllers at the origin and in the negative domain, and the sufficient condition for the system to have semiglobal fixed-time stability is also provided. Radial basis function neural networks are introduced to approximate unknown functions for solving the fixed-time control problem of unknown nonlinear pure-feedback systems, and the mean value theorem is used to solve the problem of nonaffine structure in nonlinear pure-feedback systems. The controllers designed in this paper ensure that all signals in the closedloop system are semiglobally uniform and ultimately bounded in a fixed time. Two simulation results show that appropriate design parameters can limit the tracking error within a region of the origin in a fixed time.
\end{abstract}

\section{Introduction}

Nonlinear pure-feedback systems $[1,2]$ are more common than general strict feedback nonlinear systems or nonlinear systems with input affine structure. Such systems have been studied widely in recent years because they can reflect more accurately the working conditions of actual engineering systems owing to the nonaffine structure of their control inputs and state variables.

In 1988, David S. Broomhead proposed the radial basis function (RBF) neural network [3], which is used widely in pattern recognition, signal processing, and control system theory and application because of its simple structure and generalizability. Many scholars have used it to address the uncertainties of nonlinear systems based on its approximation ability, which has been demonstrated by many researchers [4-7]. Kanellakopoulos et al. [8, 9] proposed the backstepping method and the backstepping adaptive control scheme for a class of strict feedback nonlinear systems in 1991. The adaptive control of RBF neural networks has attracted extensive attention [10-18], resulting in the stability analysis method of RBF neural networks based on the Lyapunov method [19-21]. Finite-time stability technology can be traced back to the 1960s [22], and it developed rapidly in the 1990s, owing primarily to the improvement of finitetime Lyapunov theory [23] and homogeneous systems [24]. Much has been achieved in recent years on finite-time stability [25-30]. A finite-time control system ensures that a nonlinear system converges in a finite time, but the convergence time is related to the initial state of the system. For this reason, fixed-time control systems have been proposed [31-34] that ensure that the upper limit of the fixed convergence time is no longer related to the initial state of the system but only depends on the design parameters. Since Polyakov et al. first proposed fixed-time stability control [33], nonlinear fixed-time control has developed rapidly and has been studied by many scholars. For example, in [35], a fixed-time control with generalized directional topology was proposed for nonlinear multiagent systems; in [36], a prescribed performance fixed-time recurrent neural network control was proposed for a class of uncertain nonlinear systems; in [37], a fast fixed-time nonsingular terminal sliding mode control was proposed to solve the problem of chaos suppression of power systems; and in [38], a fixedtime observer was proposed to detect distributed faults of nonlinear multiagent systems. 
In [35-40], strict feedback nonlinear systems are considered primarily, and the fixed-time control problem of more common nonlinear systems is not solved. In this paper, the fixed-time control problem is solved based on nonlinear pure-feedback systems, and the sufficient condition and design steps for semiglobal fixed-time stability are provided.

The principal contributions of this paper are as follows:

(1) The fixed-time control algorithm proposed in [35-40] does not solve the problem of the nonaffine structure of the control input $u(t)$. This paper applies fixed-time control theory in nonlinear pure-feedback systems to solve this problem.

(2) The controller designed in [40-43] has a power function similar to $z^{2 q-1}, 0<q<1$. Not selecting $q$ properly results in singularity. A novel fixed-time controller is designed in this paper to solve this problem.

(3) An RBF neural network control algorithm is introduced to approximate the unknown functions $f_{i}(\cdot)$ to overcome the difficulty of modeling accurately and solving the problem of interference in nonlinear pure-feedback systems.

The remainder of this paper is organized as follows. Section 2 presents the problem description and preliminaries. Section 3 proposes fixed-time adaptive neural tracking control using backstepping, adaptive neural networks, and Lyapunov functions for a class of unknown nonlinear pure-feedback systems to solve the problem of fixed-time tracking control for nonlinear systems with nonaffine structure. In Section 4, all signals in the closed-loop system are proved to be semiglobally uniform and ultimately bounded. In Section 5, the proposed control scheme is proved to be effective through simulation experiments. Section 6 draws conclusions.

\section{Problem Description and Preliminaries}

2.1. Problem Description. Consider the following nonlinear pure-feedback system:

$$
\left\{\begin{array}{l}
\dot{x}_{i}(t)=f_{i}\left(\bar{x}_{i}(t), x_{i+1}(t)\right)+d_{i}\left(\bar{x}_{i}, t\right), \\
\dot{x}_{n}(t)=f_{n}\left(\bar{x}_{n}(t), u(t)\right) d_{n}\left(\bar{x}_{n}, t\right), \\
y(t)=x_{1}(t),
\end{array}\right.
$$

where $\quad 1 \leq i \leq n-1, \quad \bar{x}_{i}=\left[x_{1}(t), \ldots, x_{i}(t)\right]^{T} \in R^{i} \quad$ with $i=1, \ldots, n, u(t) \in R$, and $y(t) \in R$ are state variables, system input, and system output, respectively. $f_{i}(\cdot)$ are unknown but smooth nonaffine functions, and $d_{i}(\cdot)$ are unknown but bounded disturbances.

According to the mean value theorem [44], $f_{i}\left(\bar{x}_{i}, x_{i+1}\right)=$ $f_{i}\left(\bar{x}_{i}, x_{i 0}\right)+h_{i \mu_{i}}\left(x_{i+1}-x_{i 0}\right)$ and $f_{n}\left(\bar{x}_{n}, u\right)=f_{n}\left(\bar{x}_{n}, x_{n 0}\right)+$ $h_{n \mu_{n}}\left(u-x_{n 0}\right)$, where $h_{i \mu_{i}}:=h_{i}\left(\bar{x}_{i}, x_{\mu_{i}}\right)=\partial f_{i}\left(\bar{x}_{i}, x_{\mu_{i}}\right) / \partial x_{\mu_{i}}$ with $i=1, \ldots, n, x_{\mu_{i}}=\mu_{i} x_{i+1}+\left(1-\mu_{i}\right) x_{i 0}$ with $0<\mu_{i}<1$, and $x_{i 0}$ is a known quantity at the given time $t_{0}$. System (1) can be written as

$$
\left\{\begin{array}{l}
\dot{x}_{i}=f_{i}\left(\bar{x}_{i}, x_{i 0}\right)+h_{i \mu_{i}} \cdot\left(x_{i+1}-x_{i 0}\right)+d_{i}\left(\bar{x}_{i}, t\right), \\
\dot{x}_{n}=f_{n}\left(\bar{x}_{n}, x_{n 0}\right)+h_{n \mu_{n}} \cdot\left(u-x_{n 0}\right)+d_{n}\left(\bar{x}_{n}, t\right), \\
y=x_{1} .
\end{array}\right.
$$

Remark 1. It can be seen from system (2) that the mean value theorem separates the nonaffine structure from $f_{i}(\cdot)$ in system (1).

This paper aims to design a fixed-time controller that can meet the fixed-time control requirements in nonlinear purefeedback systems, enabling the system output $y$ to track the reference signal $y_{d}$ in a fixed time. All of the signals of the closed-loop system are semiglobally uniform and ultimately bounded.

Assumption 1 (see [45]). Unknown smooth nonlinear functions $h_{i}(\cdot)$ are bounded, and there are known positive constants $b$ and $c$ with $0<b \leq\left|h_{i}(\cdot)\right|<c<\infty$, $\forall\left(\bar{x}_{i}, x_{i+1}\right) \in R^{i} \times R$. Without loss of generality, we assume that $0<b \leq h_{i}(\cdot), i=1, \ldots, n$.

For ease of calculation, vector functions are defined as $\bar{y}_{d, i}=\left[y_{d}, y_{d}^{(1)}, \ldots, y_{d}^{(i)}\right]^{T}, i=1, \ldots, n$, where $y_{d}^{(i)}$ is the $i^{\text {th }}$ derivative of $y_{d}$.

Assumption 2. Reference signal vector functions $\bar{y}_{d i}$ are known smooth continuous bounded functions. $\bar{y}_{d i} \in \Omega_{d i} \subset R^{i+1}$ with $i=1, \ldots, n$, where $\Omega_{d i}$ are known compact sets and reference signal $y_{d}$ is an $n$-order differentiable and bounded function.

\subsection{Fixed Time}

Definition 1. Consider the following nonlinear system:

$$
\dot{x}(t)=f(t, x), x(0)=x_{0},
$$

where $x \in R^{n}$ and $f: R_{+} \times R^{n} \longrightarrow R^{n}$, and assume that the origin is an equilibrium point.

Lemma 1 (see [46]). If there exist design constants $\phi_{1}>0$, $\phi_{2}>0, \alpha \in(1,+\infty)$, and $\beta \in(0,1)$ such that

$$
\dot{V}(x) \leq-\phi_{1} V^{\alpha}(x)-\phi_{2} V^{\beta}(x),
$$

where $V(x)$ is a continuous differentiable positive definite function, then system (3) is global fixed-time stable, and the fixed convergence time satisfies

$$
T \leq T_{\max }:=\frac{1}{\phi_{1}(\alpha-1)}+\frac{1}{\phi_{2}(1-\beta)} .
$$

Lemma 2 (see [40]). If there exist design constants $\phi_{1}>0$, $\phi_{2}>0, \alpha \in(1, \infty), \beta \in(0,1), \tau \in(0, \infty)$, and $\Phi \in(0,1)$ such that

$$
\dot{V}(x) \leq-\phi_{1} V^{\alpha}(x)-\phi_{2} V^{\beta}(x)+\tau,
$$

then the origin of system (3) is practical fixed-time stable and the fixed-time $T$ can be estimated by 


$$
T \leq T_{\max }:=\frac{1}{\phi_{1} \oplus(\alpha-1)}+\frac{1}{\phi_{2} \bowtie(1-\beta)} .
$$

The residual set of the solution of system (3) is given by

$$
x \in\left\{V(x) \leq \min \left\{\left(\frac{\tau}{(1-\Phi) \phi_{1}}\right)^{1 / \alpha},\left(\frac{\tau}{(1-\Phi) \phi_{2}}\right)^{1 / \beta}\right\}\right\} \text {. }
$$

Lemma 3 (see [47]). Let $x_{1}, x_{2}, \ldots, x_{n} \geq 0$. Then,

$$
\begin{array}{ll}
\sum_{i=1}^{n} x_{i}^{\rho} \geq\left(\sum_{i=1}^{n} x_{i}\right)^{\rho}, & \text { if } 0<\rho \leq 1, \\
\sum_{i=1}^{n} x_{i}^{\rho} \geq n^{1-\rho}\left(\sum_{i=1}^{n} x_{i}\right)^{\rho}, & \text { if } 1<\rho \leq \infty .
\end{array}
$$

Lemma 4 (see [48]). For any variable $x \in R$ and any positive constant $\kappa$, the following relationship holds:

$$
0 \leq|x|<\kappa+\frac{x^{2}}{\sqrt{x^{2}+\kappa^{2}}} \text {. }
$$

Lemma 5. For $y \geq x>0, x, y \in R$ and any positive constant $\chi$, then the following is satisfied

$$
\frac{y}{\sqrt{y+\chi}} \geq \frac{x}{\sqrt{x+\chi}} \text {. }
$$

Proof.

$$
\begin{aligned}
& \frac{y}{\sqrt{y+\chi}}-\frac{x}{\sqrt{x+\chi}}=\frac{y+\chi}{\sqrt{y+\chi}}-\frac{x+\chi}{\sqrt{x+\chi}}-\frac{\chi}{\sqrt{y+\chi}}+\frac{\chi}{\sqrt{x+\chi}} \\
& =(\sqrt{y+\chi}-\sqrt{x+\chi})+\left(\frac{\chi}{\sqrt{x+\chi}}-\frac{\chi}{\sqrt{y+x}}\right)
\end{aligned}
$$$$
\geq 0 \text {. }
$$

2.3. Neural Network. An RBF neural network [11, 49] is applied in this paper to approximate arbitrary continuous functions. The mathematical expression of an RBF neural network is as follows:

$$
\widehat{\varphi}=W^{T} S(Z),
$$

where $W=\left[w_{1}, w_{2}, \ldots, w_{l}\right]^{T} \in R^{l}$ is the weight vector, $l>1$ is the number of nodes of the neural network, $Z \in \Omega_{Z} \subset R^{q}$ is the input of the RBF neural network, $q$ is the input dimension of the RBF neural network, $S(Z)=\left[s_{1}(Z), s_{2}(Z), \ldots, s_{l}(Z)\right]^{T} \in R^{l}$ is the basis vector function, and $s_{i}(Z)$ is the output of the $i^{\text {th }}$ node. A Gaussian function is always chosen as $s_{i}(Z)$, i.e., $s_{i}(Z)=\exp \left[-\left(Z-\xi_{i}\right)^{T}\left(Z-\xi_{i}\right) / r_{i}^{2}\right], i=1, \ldots, l$, where $r_{i}$ is the width of the base function and $\xi_{i}=\left[\xi_{i 1}, \xi_{i 2}, \ldots, \xi_{i q}\right]^{T}$ is the center of the basis function. With a sufficient number $l$ of nodes selected, an RBF neural network can approximate an arbitrary continuous function $\varphi(Z)$ in a compact set $\Omega_{Z} \in R^{q}$ with arbitrary accuracy $\varepsilon$.

$$
\varphi(Z)=W^{* T} S(Z)+\delta(Z), \quad \forall Z \in \Omega_{Z} \in R^{q},
$$

where $\delta(Z)$ is the approximation error with $|\delta(Z)| \leq \varepsilon$ and $W^{*}$ is the given ideal constant weight vector, which is defined as

$$
W^{*}=\arg \min _{W \in R^{l}} \sup _{Z \in \Omega_{Z}}\{|\varphi(Z)-\widehat{\varphi}(Z)|\} .
$$

In this paper, let $\theta_{i}=\max \left\{\left\|W_{i}^{*}\right\|^{2} / b, i=1,2, \ldots, n\right\}$ with $\tilde{\theta}_{i}=\theta_{i}-\widehat{\theta}_{i}$, where $\widehat{\theta}_{i}$ are the estimates of the unknown constants $\theta_{i}, W_{i}^{*}$ are the ideal weight vectors of the RBF neural network, $b$ is a positive design parameter, and $\|\cdot\|$ is the norm.

Remark 2. $b$ is related to Assumption 1 .

Assumption 3 (see [40]). There are unknown constants $Q_{i}$ that make $\left|\widetilde{\theta}_{i}\right| \leq Q_{i}<\infty, i=1,2, \ldots, n$.

Lemma 6 (see [50]). Consider the Gaussian function (13). $\|S(Z)\|$ has an upper bound such that

$$
\|S(Z)\| \leq \sum_{k=0}^{\infty} 3 q(k+2)^{q-1} e^{\left(-2 p^{2} k^{2}\right) / r^{2}}:=s,
$$

where $p=1 / 2\left(\min _{i \neq j}\left\|\xi_{i}-\xi_{j}\right\|\right)$.

Lemma 6 has been proved in $[50,51]$. Since $\sum_{k=0}^{\infty} 3 q(k+2)^{q-1} e^{\left(-2 p^{2} k^{2}\right) / r^{2}}$ is convergent, $s$ is a limited value. In addition, $s$ is independent of the neural network node numbers $l$ and the neural network inputs $Z$.

\section{Design of a Fixed-Time Controller}

The backstepping design coordinate transformation is as follows:

$$
\begin{aligned}
& z_{1}=x_{1}-y_{d}, \\
& z_{i}=x_{i}-\alpha_{i-1}, \quad i=2, \ldots, n,
\end{aligned}
$$

where $\alpha_{i}$ is the virtual controller of the $i^{\text {th }}$ subsystem.

An RBF neural network is used in this paper to approximate the unknown functions $\widehat{f}_{i}\left(Z_{i}\right)$ :

$$
\widehat{f}_{i}\left(Z_{i}\right)=W_{i}^{* T} S_{i}\left(Z_{i}\right)+\delta_{i}\left(Z_{i}\right),
$$

and the inequalities involved in the following text are as follows:

$$
\begin{gathered}
z_{i} \delta_{i}\left(Z_{i}\right) \leq b k_{i 3} z_{i}^{2}+\frac{\varepsilon_{i}^{2}}{4 b k_{i 3}}, \\
z_{i} W_{i}^{* T} S_{i}\left(Z_{i}\right) \leq \frac{b \theta_{i}}{2 \eta_{i}^{2}} S_{i}^{T}\left(Z_{i}\right) S_{i}\left(Z_{i}\right) z_{i}^{2}+\frac{\eta_{i}^{2}}{2},
\end{gathered}
$$




$$
\begin{aligned}
& -b k_{i 2} 2^{(1+\beta) / 2}\left(\left(\frac{1}{2} z_{i}^{2}\right)^{(1+\beta) / 2}+\left(\frac{b \widetilde{\theta}_{i}^{2}}{2 \gamma}\right)^{(1+\beta) / 2}\right) \\
& \leq-2 b k_{i 2}\left(\frac{1}{2} z_{i}^{2}+\frac{b \widetilde{\theta}_{i}^{2}}{2 \gamma}\right)^{(1+\beta) / 2}, \\
& -b k_{i 1} 2^{(1+\alpha) / 2}\left(\left(\frac{1}{2} z_{i}^{2}\right)^{(1+\alpha) / 2}+\left(\frac{b \widetilde{\theta}_{i}^{2}}{2 \gamma}\right)^{(1+\alpha) / 2}\right) \\
& \leq-b k_{i 1} 2^{(1+\alpha) / 2}\left(\frac{1}{2} z_{i}^{2}+\frac{b \widetilde{\theta}_{i}^{2}}{2 \gamma}\right)^{(1+\alpha) / 2},
\end{aligned}
$$

where $Z_{1}=\left[x_{1}, \hat{\theta}_{1}, y_{d}, \dot{y}_{d}\right] \in \Omega_{Z_{1}} \subset R^{3+1}$ and $Z_{i}=\left[x_{1}\right.$, $\left.x_{2}, \ldots, x_{i}, \widehat{\theta}_{1}, \widehat{\theta}_{2}, \ldots, \widehat{\theta}_{i}, \bar{y}_{d i}\right] \in \Omega_{Z_{i}} \subset R^{3 i+1}$ with $2 \leq i \leq n$ are input vectors and $\eta_{i}, k_{i 1}, k_{i 2}, k_{i 3}$, and $\gamma$ are positive design parameters.

Step 1. According to $z_{1}=x_{1}-y_{d}$ and (2), we have

$$
\dot{z}_{1}=f_{1}\left(\bar{x}_{1}, x_{10}\right)+h_{1 \mu_{1}}\left(x_{2}-x_{10}\right)+d_{1}\left(\bar{x}_{1}, t\right)-\dot{y}_{d} .
$$

Construct a Lyapunov function as

$$
V_{1}=\frac{1}{2} z_{1}^{2}+\frac{b \widetilde{\theta}_{1}^{2}}{2 \gamma}
$$

Time differentiation of $V_{1}$ yields

$$
\dot{V}_{1}=z_{1} f_{1}\left(\bar{x}_{1}, x_{10}\right)+z_{1} h_{1 \mu_{1}}\left(x_{2}-x_{10}\right)+z_{1} d_{1}-z_{1} \dot{y}_{d}-\frac{b \widetilde{\theta}_{1} \dot{\hat{\theta}}_{1}}{\gamma} \text {. }
$$

Substituting $z_{2}=x_{2}-\alpha_{1}$ into (25) yields

$$
\begin{aligned}
\dot{V}_{1}= & z_{1} f_{1}\left(\bar{x}_{1}, x_{10}\right)+z_{1} h_{1 \mu_{1}} z_{2}+z_{1} h_{1 \mu_{1}} \alpha_{1}-z_{1} h_{1 \mu_{1}} x_{10} \\
& +z_{1} d_{1}-z_{1} \dot{y}_{d}-\frac{b \widetilde{\theta}_{1} \dot{\hat{\theta}}_{1}}{\gamma} .
\end{aligned}
$$

The virtual controller $\alpha_{1}$ is defined as

$$
\begin{aligned}
\alpha_{1}= & -k_{11} S_{z_{1}, 1}-k_{12} S_{z_{1}, 2}-\frac{\hat{\theta}_{1}}{2 \eta_{1}^{2}} S_{1}^{T}\left(Z_{1}\right) S_{1}\left(Z_{1}\right) z_{1} \\
& -k_{13} z_{1}+x_{10},
\end{aligned}
$$

where $k_{11}, k_{12}, k_{13}$, and $\eta_{1}$ are positive design parameters. In (27), $S_{z_{1}, 1}$ and $S_{z_{1}, 2}$ are defined as

$$
\begin{aligned}
& S_{z_{1}, 1}= \begin{cases}\frac{\left(z_{1}^{2}\right)^{(\alpha+1) / 2}}{z_{1}}, & \left|z_{1}\right| \geq \varepsilon_{10}, \\
\sum_{j=1}^{n} a_{j}\left(z_{1}^{2}\right)^{j}\left(\varepsilon_{10}^{2}\right)^{-j+(\alpha+1) / 2}, & \left|z_{1}\right|<\varepsilon_{10},\end{cases} \\
& S_{z_{1}, 2}= \begin{cases}\frac{\left(z_{1}^{2}\right)^{(\beta+1) / 2}}{z_{1}}, & \left|z_{1}\right| \geq \varepsilon_{10}, \\
\sum_{j=1}^{n} a_{j}\left(z_{1}^{2}\right)^{j}\left(\varepsilon_{10}^{2}\right)^{-j+(\beta+1) / 2}, & \left|z_{1}\right|<\varepsilon_{10},\end{cases}
\end{aligned}
$$

where $\varepsilon_{10}$ is a positive design parameter and coefficients $a_{j}$, $j=1,2, \ldots, n$, are calculated using the following equation:

$$
\left[\begin{array}{ccccc}
1 & 1 & \cdots & 1 & 1 \\
1 & 2 & \cdots & n-1 & n \\
0 & 2 \times 1 & \cdots & (n-1)(n-2) & n(n-1) \\
\vdots & \vdots & \ddots & \vdots & \vdots \\
0 & 0 & \cdots & \prod_{j=0}^{n-2}(n-1-j) & \prod_{j=0}^{n-2}(n-j)
\end{array}\right] \times\left[\begin{array}{c}
a_{1} \\
a_{2} \\
a_{3} \\
\vdots \\
a_{n}
\end{array}\right]=\left[\begin{array}{c}
b_{1} \\
b_{2} \\
b_{3} \\
\vdots \\
b_{n}
\end{array}\right],
$$


where $b_{1}=1, \quad b_{2}=3 / 4, \quad b_{3}=(3 / 4)((3 / 4)-1), \ldots$, and $b_{n}=\prod_{j=0}^{n-2}((3 / 4)-j)$.

Remark 3. One of the main contributions of this paper is to design suitable virtual controllers $\alpha_{i}$ so that nonlinear purefeedback systems meet the requirement of fixed-time control. In [40-43], virtual controllers designed exhibit similar power functions $z^{2 \alpha-1}$, where $0<\alpha<1$. If $\alpha$ is not appropriately selected, it will make $z$ unsolvable at the origin and in the negative domain. As $0<\alpha<1$, power exponents $2 \alpha-1 \in(-1,1)$, which leads to the possibility of negative power exponents. For example, if $2 \alpha-1=-1 / 3, z^{-1 / 3}$ is unsolvable at $z=0$, that is, $(0)^{-1 / 3}$ does not exist. Suppose $2 \alpha-1=q_{1} / q_{2} \in(-1,1)$, where $q_{2}$ is an even number; then, $z^{q_{1} / q_{2}}$ is unsolvable at the negative domain. For example, if $q_{1} / q_{2}=1 / 2, z^{1 / 2}$ is unsolvable at the negative domain, that is, $(-1)^{1 / 2}$ does not exist. The controller designed in this paper overcomes the aforementioned defect and promotes the application of fixed-time control in more common nonlinear systems.

Substituting $\alpha_{1}$ into (26) yields

$$
\begin{aligned}
\dot{V}_{1} \leq & z_{1} \widehat{f}_{1}\left(Z_{1}\right)+z_{1} h_{1 \mu_{1}} z_{2}-\frac{b \widetilde{\theta}_{1} \dot{\hat{\theta}}_{1}}{\gamma}-b k_{11} z_{1} S_{z_{1}, 1} \\
& -b k_{12} z_{1} S_{z_{1}, 2}-\frac{b \hat{\theta}_{1}}{2 \eta_{1}^{2}} S_{1}^{T}\left(Z_{1}\right) S_{1}\left(Z_{1}\right) z_{1}^{2}-b k_{13} z_{1}^{2},
\end{aligned}
$$

where $\widehat{f}_{1}\left(Z_{1}\right)=f_{1}\left(\bar{x}_{1}, x_{10}\right)+d_{1}-\dot{y}_{d}$.

The RBF neural network (18) is utilized to approximate $\widehat{f}_{1}\left(Z_{1}\right)$ and introduce inequalities (19) and (20), following which (30) can be rewritten as

$$
\begin{aligned}
\dot{V}_{1} \leq & \frac{b \widetilde{\theta}_{1}}{\gamma}\left(\frac{\gamma}{2 \eta_{1}^{2}} S_{1}^{T}\left(Z_{1}\right) S_{1}\left(Z_{1}\right) z_{1}^{2}-\dot{\hat{\theta}}_{1}\right) \\
& +\sigma_{1}+z_{1} h_{1 \mu_{1}} z_{2}-b k_{11} z_{1} S_{z_{1}, 1}-b k_{12} z_{1} S_{z_{1}, 2},
\end{aligned}
$$

where $\sigma_{1}=\eta_{1}^{2} / 2+\varepsilon_{1}^{2} / 4 b k_{13}$.

The adaptive law $\widehat{\theta}_{1}$ is then defined as

$$
\dot{\hat{\theta}}_{1}=\frac{\gamma}{2 \eta_{1}^{2}} S_{1}^{T}\left(Z_{1}\right) S_{1}\left(Z_{1}\right) z_{1}^{2}-\lambda \widehat{\theta}_{1},
$$

where $\lambda$ is a positive design parameter.

Combining with Assumption 3 and substituting (32) into (31), one can obtain

$$
\begin{aligned}
\dot{V}_{1} \leq & -b k_{11} z_{1} S_{z_{1}, 1}-b k_{12} z_{1} S_{z_{1}, 2}-b k_{11} 2^{(1+\alpha) / 2}\left(\frac{b \widetilde{\theta}_{1}^{2}}{2 \gamma}\right)^{(1+\alpha) / 2} \\
& -b k_{12} 2^{(1+\beta) / 2}\left(\frac{b \widetilde{\theta}_{1}^{2}}{2 \gamma}\right)^{(1+\beta) / 2}+z_{1} h_{1 \mu_{1}} z_{2}+C_{1}
\end{aligned}
$$

where

$$
\begin{aligned}
\beta_{1} & =b k_{11}\left(\frac{b Q_{1}^{2}}{\gamma}\right)^{(1+\alpha) / 2}+b k_{12}\left(\frac{b Q_{1}^{2}}{\gamma}\right)^{(1+\beta) / 2}, \frac{\lambda b \tilde{\theta}_{1} \hat{\theta}_{1}}{\gamma} \\
& \leq \frac{\lambda b}{\gamma}\left(\frac{\theta_{1}^{2}}{2}-\frac{\tilde{\theta}_{1}^{2}}{2}\right) \leq \frac{\lambda b}{2 \gamma} \theta_{1}^{2},
\end{aligned}
$$

According to (21), (22), and (28), if $\left|z_{1}\right| \geq \varepsilon_{10}$, then (33) is rewritten as

$$
\begin{aligned}
\dot{V}_{1} \leq & -b k_{11} 2^{(1+\alpha) / 2}\left(\frac{1}{2} z_{1}^{2}+\frac{b \widetilde{\theta}_{1}^{2}}{2 \gamma}\right)^{(1+\alpha) / 2} \\
& -2 b k_{12}\left(\frac{1}{2} z_{1}^{2}+\frac{b \widetilde{\theta}_{1}^{2}}{2 \gamma}\right)^{(1+\beta) / 2}+z_{1} h_{1 \mu_{1}} z_{2}+C_{1} .
\end{aligned}
$$

According to (21), (22), and (28), if $\left|z_{1}\right|<\varepsilon_{10}$, then (33) is rewritten as

$$
\begin{aligned}
\dot{V}_{1} \leq & b k_{11}\left(z_{1}^{2}\right)^{(1+\alpha) / 2}-b k_{11} z_{1} \sum_{j=1}^{n} a_{j}\left(z_{1}^{2}\right)^{j}\left(\varepsilon_{10}^{2}\right)^{-j+((1+\alpha) / 2)} \\
& +b k_{12}\left(z_{1}^{2}\right)^{(1+\beta) / 2}-b k_{12} z_{1} \sum_{j=1}^{n} a_{j}\left(z_{1}^{2}\right)^{j}\left(\varepsilon_{10}^{2}\right)^{-j+((1+\beta) / 2)} \\
& -b k_{11} 2^{(1+\alpha) / 2}\left(\frac{1}{2} z_{1}^{2}+\frac{b \widetilde{\theta}_{1}^{2}}{2 \gamma}\right)^{(1+\alpha) / 2} \\
& -2 b k_{12}\left(\frac{1}{2} z_{1}^{2}+\frac{b \widetilde{\theta}_{1}^{2}}{2 \gamma}\right)^{(1+\beta) / 2}+z_{1} h_{1 \mu_{1}} z_{2}+C_{1} .
\end{aligned}
$$

Remark 4 (see [52]). Based on (28), when $\left|z_{1}\right|<\varepsilon_{10}$, there is an additional term in (36): $b k_{11}\left(z_{1}^{2}\right)^{(1+\alpha) / 2}-b k_{11} z_{1}$ $\sum_{j=1}^{n} a_{j}\left(z_{1}^{2}\right)^{j}\left(\varepsilon_{10}^{2}\right)^{-j+((1+\alpha) / 2)}+b k_{12}\left(z_{1}^{2}\right)^{(1+\beta) / 2}-b k_{12} z_{1} \sum_{j=1}^{n}$ $a_{j}\left(z_{1}^{2}\right)^{j}\left(\varepsilon_{10}^{2}\right)^{-j+((1+\beta) / 2)}$. Note that if $\left|z_{1}\right|<\varepsilon_{10}$, then this additional term is obviously limited by some smaller constant $\varepsilon_{11}$, so the structure of (35) is retained, while the constant term $C_{1}$ only slightly increases. Owing to page limitations and to avoid repetitive discussions, we will omit this part in the rest of the analysis.

Step 2. According to $z_{2}=x_{2}-\alpha_{1}$, we have

$$
\dot{z}_{2}=f_{2}\left(\bar{x}_{2}, x_{20}\right)+h_{2 \mu_{2}}\left(x_{3}-x_{20}\right)-\dot{\alpha}_{1}+d_{2}\left(\bar{x}_{2}, t\right),
$$

where

$$
\dot{\alpha}_{1}=\frac{\partial \alpha_{1}}{\partial x_{1}} \dot{x}_{1}+\frac{\partial \alpha_{1}}{\partial y_{d}} \dot{y}_{d}+\frac{\partial \alpha_{1}}{\partial \widehat{\hat{\theta}}_{1}} .
$$

Construct a Lyapunov function as

$$
V_{2}=V_{1}+\frac{1}{2} z_{2}^{2}+\frac{b \tilde{\theta}_{2}^{2}}{2 \gamma}
$$

The derivative of $V_{2}$ is written as 


$$
\begin{aligned}
\dot{V}_{2} \leq & -b k_{11} 2^{(1+\alpha) / 2}\left(\frac{1}{2} z_{1}^{2}+\frac{b \widetilde{\theta}_{1}^{2}}{2 \gamma}\right)^{(1+\alpha) / 2} \\
& -2 b k_{12}\left(\frac{1}{2} z_{1}^{2}+\frac{b \widetilde{\theta}_{1}^{2}}{2 \gamma}\right)^{(1+\beta) / 2}+z_{2} \widehat{f}_{2}\left(Z_{2}\right)+z_{2} h_{2 \mu_{2}} z_{3} \\
& +z_{2} h_{2 \mu_{2}} \alpha_{2}-z_{2} h_{2 \mu_{2}} x_{20}-z_{2} \frac{\partial \alpha_{1} \dot{\hat{\theta}}_{1}}{\partial \widehat{\theta}_{1}}-\frac{b \tilde{\theta}_{2} \dot{\hat{\theta}}_{2}}{\gamma}+z_{2} M_{1}\left(Z_{2}\right)+C_{1},
\end{aligned}
$$

where

$$
\widehat{f}_{2}\left(Z_{2}\right)=f_{2}\left(\bar{x}_{2}, x_{20}\right)-\frac{\partial \alpha_{1}}{\partial x_{1}} \dot{x}_{1}-\frac{\partial \alpha_{1}}{\partial y_{d}} \dot{y}_{d}+z_{1} h_{1 \mu_{1}}+d_{2}-M_{1}\left(Z_{2}\right) \text {. }
$$

Remark 5. $M_{1}\left(Z_{2}\right)$ is a smooth function that is used to overcome the design difficulty of $\widehat{\theta}_{1} \partial \alpha_{1} / \partial \widehat{\theta}_{1}$.

The virtual controller $\alpha_{2}$ is defined as

$$
\alpha_{2}=-k_{21} S_{z_{2}, 1}-k_{22} S_{z_{2}, 2}-\frac{\widehat{\theta}_{2}}{2 \eta_{2}^{2}} S_{2}^{T}\left(Z_{2}\right) S_{2}\left(Z_{2}\right) z_{2}-k_{23} z_{2}+x_{20}
$$

where $k_{21}, k_{22}, k_{23}$, and $\eta_{2}$ are positive design parameters.

In (42), $S_{z_{2}, 1}$ and $S_{z_{2}, 2}$ are defined as

$$
\begin{aligned}
S_{z_{2}, 1}= \begin{cases}\frac{\left(z_{2}^{2}\right)^{(\alpha+1) / 2}}{z_{2}}, & \left|z_{2}\right| \geq \varepsilon_{20}, \\
\sum_{j=1}^{n} a_{j}\left(z_{2}^{2}\right)^{j}\left(\varepsilon_{20}^{2}\right)^{-j+((\alpha+1) / 2)}, & \left|z_{2}\right|<\varepsilon_{20},\end{cases} \\
S_{z_{2}, 2}= \begin{cases}\frac{\left(z_{2}^{2}\right)^{(\beta+1) / 2}}{z_{2}}, & \left|z_{2}\right| \geq \varepsilon_{20}, \\
\sum_{j=1}^{n} a_{j}\left(z_{2}^{2}\right)^{j}\left(\varepsilon_{20}^{2}\right)^{-j+((\beta+1) / 2)}, & \left|z_{2}\right|<\varepsilon_{20},\end{cases}
\end{aligned}
$$

where $\varepsilon_{20}$ is a positive design parameter.

Choose the adaptive law $\widehat{\theta}_{2}$ as

$$
\dot{\hat{\theta}}_{2}=\frac{\gamma}{2 \eta_{2}^{2}} S_{2}^{T}\left(Z_{2}\right) S_{2}\left(Z_{2}\right) z_{2}^{2}-\lambda \widehat{\theta}_{2} \text {. }
$$

Combining (18)-(20) and Assumption 3, substituting (42) and (44) into (40), and adopting the same design method as in Step 1 yields (45) as a rewriting of (40).

$$
\begin{aligned}
\dot{V}_{2} \leq & -b k_{21} z_{2} S_{z_{2}, 1}-b k_{21} 2^{(1+\alpha) / 2}\left(\frac{b \widetilde{\theta}_{2}^{2}}{2 \gamma}\right)^{(1+\alpha) / 2} \\
& -b k_{22} z_{2} S_{z_{2}, 2}-b k_{22} 2^{(1+\beta) / 2}\left(\frac{b \widetilde{\theta}_{2}^{2}}{2 \gamma}\right)^{(1+\beta) / 2} \\
& -b k_{11} 2^{(1+\alpha) / 2}\left(\frac{1}{2} z_{1}^{2}+\frac{b \widetilde{\theta}_{1}^{2}}{2 \gamma}\right)^{(1+\alpha) / 2} \\
& -2 b k_{12}\left(\frac{1}{2} z_{1}^{2}+\frac{b \widetilde{\theta}_{1}^{2}}{2 \gamma}\right)^{(1+\beta) / 2} \\
& +\sum_{j=1}^{2} C_{j}+z_{2} h_{2 \mu_{2}} z_{3}+z_{2}\left(M_{1}\left(Z_{2}\right)-\frac{\left.\partial \alpha_{1} \dot{\hat{\theta}}_{1}\right)}{\partial \widehat{\theta}_{1}}\right.
\end{aligned}
$$

where

$$
\begin{aligned}
\frac{\lambda b \widetilde{\theta}_{2} \hat{\theta}_{2}}{\gamma} & \leq \frac{\lambda b}{\gamma}\left(\frac{\theta_{2}^{2}}{2}-\frac{\tilde{\theta}_{2}^{2}}{2}\right) \leq \frac{\lambda b}{2 \gamma} \theta_{2}^{2}, \\
\beta_{2} & =b k_{21}\left(\frac{b Q_{2}^{2}}{\gamma}\right)^{(1+\alpha) / 2}+b k_{22}\left(\frac{b Q_{2}^{2}}{\gamma}\right)^{(1+\beta) / 2} \\
\sigma_{2} & =\frac{\eta_{2}^{2}}{2}+\frac{\varepsilon_{2}^{2}}{4 b k_{23}} \\
C_{2} & =\sigma_{2}+\beta_{2}+\frac{\lambda b \theta_{2}^{2}}{2 \gamma} .
\end{aligned}
$$

According to (21) and (22) and Remark 4, we consider only $\left|z_{2}\right| \geq \varepsilon_{20}$. Then, (45) can be written as

$$
\begin{aligned}
\dot{V}_{2} \leq & -b 2^{(1+\alpha) / 2} \sum_{j=1}^{2} k_{j 1}\left(\frac{1}{2} z_{j}^{2}+\frac{b \widetilde{\theta}_{j}^{2}}{2 \gamma}\right)^{(1+\alpha) / 2} \\
& -2 b \sum_{j=1}^{2} k_{j 2}\left(\frac{1}{2} z_{j}^{2}+\frac{b \widetilde{\theta}_{j}^{2}}{2 \gamma}\right)^{(1+\beta) / 2} \\
& +\sum_{j=1}^{2} C_{j}+z_{2} h_{2 \mu_{2}} z_{3}+z_{2}\left(M_{1}\left(Z_{2}\right)-\frac{\partial \alpha_{1} \dot{\hat{\theta}}_{1}}{\partial \widehat{\theta}_{1}}\right) .
\end{aligned}
$$

It can be seen from (47) that defining the design smooth function $M_{1}\left(Z_{2}\right)$ to overcome the design difficulty of $\left(\partial \alpha_{1} / \partial \widehat{\theta}_{1}\right) \widehat{\theta}_{1}$ is one of the difficulties of designing the controllers in this paper.

From Lemmas 4-6 and (32), it follows that 


$$
\begin{aligned}
-z_{2} \frac{\partial \alpha_{1}}{\partial \widehat{\theta}_{1}} \dot{\hat{\theta}}_{1} \leq & -z_{2} \frac{\partial \alpha_{1}}{\partial \widehat{\theta}_{1}}\left(\frac{\gamma}{2 \eta_{1}^{2}} S_{1}^{T}\left(Z_{1}\right) S_{1}\left(Z_{1}\right) z_{1}^{2}-\lambda \widehat{\theta}_{1}\right) \\
\leq & \frac{z_{2}^{2}\left(\partial \alpha_{1} / \partial \widehat{\theta}_{1}\right)^{2}\left(\left(\gamma / 2 \eta_{1}^{2}\right) S_{1}^{T}\left(Z_{1}\right) S_{1}\left(Z_{1}\right) z_{1}^{2}\right)^{2}}{\sqrt{z_{2}^{2}\left(\partial \alpha_{1} / \partial \widehat{\theta}_{1}\right)^{2}\left(\left(\gamma / 2 \eta_{1}^{2}\right) S_{1}^{T}\left(Z_{1}\right) S_{1}\left(Z_{1}\right) z_{1}^{2}\right)^{2}+\varsigma_{2,1}^{2}}} \\
& +z_{2} \frac{\partial \alpha_{1}}{\partial \widehat{\theta}_{1}} \lambda \widehat{\theta}_{1} \\
\leq & \frac{z_{2}^{2}\left(\partial \alpha_{1} / \partial \widehat{\theta}_{1}\right)^{2}\left(\left(\gamma / 2 \eta_{1}^{2}\right) s^{2} z_{1}^{2}\right)^{2}}{\sqrt{z_{2}^{2}\left(\partial \alpha_{1} / \partial \hat{\theta}_{1}\right)^{2}\left(\left(\gamma / 2 \eta_{1}^{2}\right) s^{2} z_{1}^{2}\right)^{2}+\varsigma_{2,1}^{2}}}+z_{2} \frac{\partial \alpha_{1}}{\partial \widehat{\theta}_{1}} \lambda \widehat{\theta}_{1} .
\end{aligned}
$$

Therefore, $M_{1}\left(Z_{2}\right)$ can be defined as

$$
M_{1}\left(Z_{2}\right)=-\lambda \frac{\partial \alpha_{1}}{\partial \widehat{\theta}_{1}} \widehat{\theta}_{1}-\frac{z_{2}\left(\partial \alpha_{1} / \partial \widehat{\theta}_{1}\right)^{2}\left(\left(\gamma / 2 \eta_{1}^{2}\right) s^{2} z_{1}^{2}\right)^{2}}{\sqrt{z_{2}^{2}\left(\partial \alpha_{1} / \partial \widehat{\theta}_{1}\right)^{2}\left(\left(\gamma / 2 \eta_{1}^{2}\right) s^{2} z_{1}^{2}\right)^{2}+\varsigma_{2,1}^{2}}}
$$

with the result that

$$
z_{2}\left(M_{1}\left(Z_{2}\right)-\frac{\partial \alpha_{1}}{\partial \hat{\theta}_{1}}\right) \leq 0
$$

Substituting (50) into (47) yields

$$
\begin{aligned}
\dot{V}_{2} \leq & -b 2^{(1+\alpha) / 2} \sum_{j=1}^{2} k_{j 1}\left(\frac{1}{2} z_{j}^{2}+\frac{b \widetilde{\theta}_{j}^{2}}{2 \gamma}\right)^{(1+\alpha) / 2} \\
& -2 b \sum_{j=1}^{2} k_{j 2}\left(\frac{1}{2} z_{j}^{2}+\frac{b \widetilde{\theta}_{j}^{2}}{2 \gamma}\right)^{(1+\beta) / 2}+\sum_{j=1}^{2} C_{j}+z_{2} h_{2 \mu_{2}} z_{3} .
\end{aligned}
$$

Step 3. $(3 \leq k \leq n-1)$. According to $z_{k}=x_{k}-\alpha_{k-1}$, we have

$$
\dot{z}_{k}=f_{k}\left(\bar{x}_{k}, x_{k 0}\right)+h_{k \mu_{k}}\left(x_{k+1}-x_{k 0}\right)+d_{k}\left(\bar{x}_{k}, t\right)-\dot{\alpha}_{k-1} \text {, }
$$

where

$$
\dot{\alpha}_{k-1}=\sum_{j=1}^{k-1} \frac{\partial \alpha_{k-1}}{\partial x_{j}} \dot{x}_{j}+\frac{\partial \alpha_{k-1}}{\partial \bar{y}_{d, k-1}} \dot{\bar{y}}_{d . k-1}+\sum_{j=1}^{k-1} \frac{\partial \alpha_{k-1}}{\partial \widehat{\theta}_{j}} \dot{\hat{\theta}}_{j} .
$$

$$
\text { Constructing a Lyapunov function as }
$$
$V_{k}=V_{k-1}+\left(z_{k}^{2} / 2\right)+\left(b \tilde{\theta}_{k}^{2} / 2 \gamma\right)$ yields

$$
\begin{aligned}
\dot{V}_{k} \leq & -b 2^{(1+\alpha) / 2} \sum_{j=1}^{k-1} k_{j 1}\left(\frac{1}{2} z_{j}^{2}+\frac{b \widetilde{\theta}_{j}^{2}}{2 \gamma}\right)^{(1+\alpha) / 2} \\
& -2 b \sum_{j=1}^{k-1} k_{j 2}\left(\frac{1}{2} z_{j}^{2}+\frac{b \widetilde{\theta}_{j}^{2}}{2 \gamma}\right)^{(1+\beta) / 2}+\sum_{j=1}^{k-1} C_{j}+z_{k} \widehat{f}_{k}\left(Z_{k}\right) \\
& +z_{k} h_{k \mu_{k}} z_{k+1}+z_{k} h_{k \mu_{k}} \alpha_{k}-z_{k} h_{k \mu_{k}} x_{k 0}-z_{k} \sum_{j=1}^{k-1} \frac{\partial \alpha_{k-1}}{\partial \widehat{\theta}_{j}} \dot{\hat{\theta}}_{j} \\
& -\frac{b \widetilde{\theta}_{k} \dot{\hat{\theta}}_{k}}{\gamma}+z_{k} M_{k-1}\left(Z_{k}\right),
\end{aligned}
$$

where

$$
\begin{aligned}
\widehat{f}_{k}\left(Z_{k}\right)= & f_{k}\left(\bar{x}_{k}, x_{k 0}\right)-\sum_{j=1}^{k-1} \frac{\partial \alpha_{k-1}}{\partial x_{j}} \dot{x}_{j}-\frac{\partial \alpha_{k-1}}{\partial y_{d, k-1}} \dot{\bar{y}}_{d, k-1} \\
& +z_{k-1} h_{k-1 \mu_{k-1}}+d_{k}\left(\bar{x}_{k}, t\right)-M_{k-1}\left(Z_{k}\right) .
\end{aligned}
$$

Define virtual controller $\alpha_{k}$ as

$$
\alpha_{k}=-k_{k 1} S_{z_{k}, 1}-k_{k 2} S_{z_{k}, 2}-\frac{\hat{\theta}_{k}}{2 \eta_{k}} S_{k}^{T}\left(Z_{k}\right) S_{k}\left(Z_{k}\right) z_{k}-k_{k 3} z_{k}+x_{k 0},
$$

where $k_{k 1}, k_{k 2}, k_{k 3}$, and $\eta_{k}$ are positive design parameters. In (56), $S_{z_{k}, 1}$ and $S_{z_{k}, 2}$ are defined as

$$
\begin{aligned}
& S_{z_{k}, 1}= \begin{cases}\frac{\left(z_{k}^{2}\right)^{(\alpha+1) / 2}}{z_{k}}, & \left|z_{k}\right| \geq \varepsilon_{k 0}, \\
\sum_{j=1}^{n} a_{j}\left(z_{k}^{2}\right)^{j}\left(\varepsilon_{k 0}^{2}\right)^{-j+((\alpha+1) / 2)}, & \left|z_{k}\right|<\varepsilon_{k 0},\end{cases} \\
& S_{z_{k}, 2}= \begin{cases}\frac{\left(z_{k}^{2}\right)^{(\beta+1) / 2}}{z_{k}}, & \left|z_{k}\right| \geq \varepsilon_{k 0}, \\
\sum_{j=1}^{n} a_{j}\left(z_{k}^{2}\right)^{j}\left(\varepsilon_{k 0}^{2}\right)^{-j+((\beta+1) / 2)}, & \left|z_{k}\right|<\varepsilon_{k 0},\end{cases}
\end{aligned}
$$

where $\varepsilon_{k 0}$ is a positive design parameter.

Define the adaptive law $\widehat{\theta}_{k}$ as

$$
\dot{\hat{\theta}}_{k}=\frac{\gamma}{2 \eta_{k}^{2}} S_{k}^{T}\left(Z_{k}\right) S_{k}\left(Z_{k}\right) z_{k}^{2}-\lambda \widehat{\theta}_{k} .
$$

Combining (18)-(20) and Assumption 3 and substituting (56) and (58) into (54) enable (54) to be rewritten as 


$$
\begin{aligned}
\dot{V}_{k} \leq & -b 2^{(1+\alpha) / 2} \sum_{j=1}^{k-1} k_{j 1}\left(\frac{1}{2} z_{j}^{2}+\frac{b \widetilde{\theta}_{j}^{2}}{2 \gamma}\right)^{(1+\alpha) / 2} \\
& -b k_{k 1} z_{k} S_{z_{k}, 1}-b k_{k 1} 2^{(1+\alpha) / 2}\left(\frac{b \widetilde{\theta}_{k}^{2}}{2 \gamma}\right)^{(1+\alpha) / 2} \\
& -2 b \sum_{j=1}^{k-1} k_{j 1}\left(\frac{1}{2} z_{j}^{2}+\frac{b \widetilde{\theta}_{j}^{2}}{2 \gamma}\right)^{(1+\beta) / 2} \\
& -b k_{k 2} z_{k} S_{z_{k}, 2}-b k_{k 2} 2^{(1+\beta) / 2}\left(\frac{b \widetilde{\theta}_{k}^{2}}{2 \gamma}\right)^{(1+\beta) / 2} \\
& +\sum_{j=1}^{k} C_{j}+z_{k} h_{k \mu_{k}} z_{k+1}+z_{k}\left(M_{k-1}\left(Z_{k}\right)-\sum_{j=1}^{k-1} \frac{\partial \alpha_{k-1}}{\partial \widehat{\theta}_{j}}\right)
\end{aligned}
$$

where

$$
\begin{aligned}
\frac{\lambda b \widetilde{\theta}_{k} \hat{\theta}_{k}}{\gamma} \leq \frac{\lambda b}{\gamma}\left(\frac{\theta_{k}^{2}}{2}-\frac{\tilde{\theta}_{k}^{2}}{2}\right) \leq \frac{\lambda b}{2 \gamma} \theta_{k}^{2}, \\
\beta_{k}=b k_{k 1}\left(\frac{b Q_{k}^{2}}{\gamma}\right)^{(1+\alpha) / 2}+b k_{k 2}\left(\frac{b Q_{k}^{2}}{\gamma}\right)^{(1+\beta) / 2}, \\
\sigma_{k}=\frac{\eta_{k}^{2}}{2}+\frac{\varepsilon_{k}^{2}}{4 b k_{k 3}}, \\
C_{k}=\sigma_{k}+\beta_{k}+\frac{\lambda b \theta_{k}^{2}}{2 \gamma} .
\end{aligned}
$$

In accordance with (21) and (22) and Remark 4, we consider only $\left|z_{k}\right| \geq \varepsilon_{k 0}$. Then, (59) can be written as

$$
\begin{aligned}
\dot{V}_{k} \leq & -b 2^{(1+\alpha) / 2} \sum_{j=1}^{k} k_{j 1}\left(\frac{1}{2} z_{j}^{2}+\frac{b \tilde{\theta}_{j}^{2}}{2 \gamma}\right)^{(1+\alpha) / 2} \\
& -2 b \sum_{j=1}^{k} k_{j 2}\left(\frac{1}{2} z_{j}^{2}+\frac{b \widetilde{\theta}_{j}^{2}}{2 \gamma}\right)+\sum_{j=1}^{k} C_{j} \\
& +z_{k} h_{k \mu_{k}} z_{k+1}+z_{k}\left(M_{k-1}\left(Z_{k}\right)-\sum_{j=1}^{k-1} \frac{\partial \alpha_{k-1}}{\partial \widehat{\hat{\theta}}_{j}}\right) .
\end{aligned}
$$

Lemmas 4-6 and (58) yield

$$
\begin{aligned}
-z_{k} \sum_{j=1}^{k-1} \frac{\partial \alpha_{k-1}}{\partial \hat{\theta}_{j}} & \leq-z_{k} \sum_{j=1}^{k-1} \frac{\partial \alpha_{k-1}}{\partial \widehat{\theta}_{j}}\left(\frac{\gamma}{2 \eta_{j}^{2}} S_{j}^{T}\left(Z_{j}\right) S_{j}\left(Z_{j}\right) z_{j}^{2}-\lambda \widehat{\theta}_{j}\right) \\
\leq & \sum_{j=1}^{k-1} \frac{z_{k}^{2}\left(\partial \alpha_{k-1} / \partial \widehat{\theta}_{j}\right)^{2}\left(\left(\gamma / 2 \eta_{j}^{2}\right) s^{2} z_{j}^{2}\right)}{\sqrt{z_{k}^{2}\left(\partial \alpha_{k-1} / \partial \widehat{\theta}_{j}\right)^{2}\left(\left(\gamma / 2 \eta_{j}^{2}\right) s^{2} z_{j}^{2}\right)^{2}+\varsigma_{k, j}^{2}}} \\
& +\lambda z_{k} \sum_{j=1}^{k-1} \frac{\partial \alpha_{k-1} \widehat{\theta}_{j} .}{\partial \widehat{\theta}_{j}}
\end{aligned}
$$
as

$$
\begin{aligned}
M_{k-1}\left(Z_{k}\right)= & -\sum_{j=1}^{k-1} \frac{z_{k}\left(\partial \alpha_{k-1} / \partial \widehat{\theta}_{j}\right)^{2}\left(\left(\gamma / 2 \eta_{j}^{2}\right) s^{2} z_{j}^{2}\right)^{2}}{\sqrt{z_{k}^{2}\left(\partial \alpha_{k-1} / \partial \hat{\theta}_{j}\right)^{2}\left(\left(\gamma / 2 \eta_{j}^{2}\right) s^{2} z_{j}^{2}\right)^{2}+\varsigma_{k, j}^{2}}} \\
& -\lambda \sum_{j=1}^{k-1} \frac{\partial \alpha_{k-1} \widehat{\theta}_{j}}{\partial \widehat{\theta}_{j}}
\end{aligned}
$$

with the result that

$$
z_{k}\left(M_{k-1}\left(Z_{k}\right)-\sum_{j=1}^{k-1} \frac{\partial \alpha_{k-1} \dot{\widehat{\theta}}_{j}}{\partial \widehat{\theta}_{j}}\right) \leq 0
$$

Substituting (64) into (61) yields

$$
\begin{aligned}
\dot{V}_{k} \leq & -b 2^{(1+\alpha) / 2} \sum_{j=1}^{k} k_{j 1}\left(\frac{1}{2} z_{j}^{2}+\frac{b \widetilde{\theta}_{j}^{2}}{2 \gamma}\right)^{(1+\alpha) / 2} \\
& -2 b \sum_{j=1}^{k} k_{j 2}\left(\frac{1}{2} z_{j}^{2}+\frac{b \widetilde{\theta}_{j}^{2}}{2 \gamma}\right)^{(1+\beta) / 2}+\sum_{j=1}^{k} C_{j}+z_{k} h_{k \mu_{k}} z_{k+1} .
\end{aligned}
$$

Step 4. According to $z_{n}=x_{n}-\alpha_{n-1}$, we have

$$
\dot{z}_{n}=f_{n}\left(\bar{x}_{n}, x_{n 0}\right)+h_{n \mu_{n}}\left(u-x_{n 0}\right)+d_{n}\left(\bar{x}_{n}, t\right)-\dot{\alpha}_{n-1},
$$

where

$$
\dot{\alpha}_{n-1}=\sum_{j=1}^{n-1} \frac{\partial \alpha_{n-1}}{\partial x_{j}} \dot{x}_{j}+\frac{\partial \alpha_{n-1}}{\partial \bar{y}_{d, n-1}} \dot{\bar{y}}_{d \cdot n-1}+\sum_{j=1}^{n-1} \frac{\partial \alpha_{n-1}}{\partial \widehat{\hat{\theta}}_{j}} \dot{\hat{\theta}}_{j} .
$$

Constructing a Lyapunov function as $V_{n}=V_{n-1}+z_{n}^{2} / 2+$ $b \widetilde{\theta}_{n}^{2} / 2 \gamma$ yields 


$$
\begin{aligned}
\dot{V}_{n} \leq & -b 2^{(1+\alpha) / 2} \sum_{j=1}^{n-1} k_{j 1}\left(\frac{1}{2} z_{j}^{2}+\frac{b \widetilde{\theta}_{j}^{2}}{2 \gamma}\right)^{(1+\alpha) / 2} \\
& -2 b \sum_{j=1}^{n-1} k_{j 2}\left(\frac{1}{2} z_{j}^{2}+\frac{b \widetilde{\theta}_{j}^{2}}{2 \gamma}\right)^{(1+\beta) / 2}+\sum_{j=1}^{n-1} C_{j} \\
& +z_{n} \widehat{f}_{n}\left(Z_{n}\right)+z_{n} h_{n \mu_{n}} u-z_{n} h_{n \mu_{n}} x_{n 0}-z_{n} \sum_{j=1}^{n-1} \frac{\partial \alpha_{n-1}}{\partial \widehat{\hat{\theta}}_{j}} \\
& -\frac{b \tilde{\theta}_{n}}{\gamma} \dot{\hat{\theta}}_{n}
\end{aligned}
$$

where

$$
\begin{aligned}
\widehat{f}_{n}\left(Z_{n}\right)= & f_{n}\left(\bar{x}_{n}, x_{n 0}\right)-\sum_{j=1}^{n-1} \frac{\partial \alpha_{n-1}}{\partial x_{j}} \dot{x}_{j}-\frac{\partial \alpha_{n-1}}{\partial \bar{y}_{d, n-1}} \dot{\bar{y}}_{d, n-1} \\
& +z_{n-1} h_{n-1 \mu_{n-1}}+d_{n}\left(\bar{x}_{n}, t\right)-M_{n-1}\left(Z_{n}\right) .
\end{aligned}
$$

Define an actual controller $u$ as

$$
\begin{aligned}
u= & -k_{n 1} S_{z_{n}, 1}-k_{n 2} S_{z_{n}, 2}-\frac{\widehat{\theta}_{n}}{2 \eta_{n}^{2}} S_{n}^{T}\left(Z_{n}\right) S_{n}\left(Z_{n}\right) z_{n} \\
& -k_{n 3} z_{n}+x_{n 0},
\end{aligned}
$$

where $k_{n 1}, k_{n 2}, k_{n 3}$, and $\eta_{n}$ are positive design parameters. In (70), $S_{z_{n}, 1}$ and $S_{z_{n}, 2}$ are defined as

$$
\begin{aligned}
& S_{z_{n}, 1}= \begin{cases}\frac{\left(z_{n}^{2}\right)^{(\alpha+1) / 2}}{z_{n}}, & \left|z_{n}\right| \geq \varepsilon_{n 0}, \\
\sum_{j=1}^{n} a_{j}\left(z_{n}^{2}\right)^{j}\left(\varepsilon_{n 0}^{2}\right)^{-j+((\alpha+1) / 2)}, & \left|z_{n}\right|<\varepsilon_{n 0},\end{cases} \\
& S_{z_{n}, 2}= \begin{cases}\frac{\left(z_{n}^{2}\right)^{(\beta+1) / 2}}{z_{n}}, & \left|z_{n}\right| \geq \varepsilon_{n 0}, \\
\sum_{j=1}^{n} a_{j}\left(z_{n}^{2}\right)^{j}\left(\varepsilon_{n 0}^{2}\right)^{-j+((\beta+1) / 2)}, & \left|z_{n}\right|<\varepsilon_{n 0},\end{cases}
\end{aligned}
$$

where $\varepsilon_{n 0}$ is a positive design parameter.

The adaptive law $\widehat{\theta}_{n}$ is defined as

$$
\dot{\hat{\theta}}_{n}=\frac{\gamma}{2 \eta_{n}^{2}} S_{n}^{T}\left(Z_{n}\right) S_{n}\left(Z_{n}\right) z_{n}^{2}-\lambda \widehat{\theta}_{n} .
$$

Combining (18)-(20) and Assumption 3 and substituting (70) and (72) into (68) enable (68) to be rewritten as

$$
\begin{aligned}
\dot{V}_{n} \leq & -b 2^{(1+\alpha) / 2} \sum_{j=1}^{n-1} k_{j 1}\left(\frac{1}{2} z_{j}^{2}+\frac{b \widetilde{\theta}_{j}^{2}}{2 \gamma}\right)^{(1+\alpha) / 2}-b k_{n 1} z_{n} S_{z_{n}, 1} \\
& -b k_{n 1} 2^{(1+\alpha) / 2}\left(\frac{b \widetilde{\theta}_{n}^{2}}{2 \gamma}\right)^{(1+\alpha) / 2}-2 b \sum_{j=1}^{n-1} k_{j 2}\left(\frac{1}{2} z_{j}^{2}+\frac{b \widetilde{\theta}_{j}^{2}}{2 \gamma}\right)^{(1+\beta) / 2} \\
& -b k_{n 2} z_{n} S_{z_{n}, 2}-b k_{n 2} 2^{(1+\beta) / 2}\left(\frac{b \widetilde{\theta}_{n}^{2}}{2 \gamma}\right)^{(1+\beta) / 2} \\
& +\sum_{j=1}^{n} C_{j}+z_{n}\left(M_{n-1}\left(Z_{n}\right)-\sum_{j=1}^{n-1} \frac{\partial \alpha_{k-1}}{\partial \hat{\theta}_{j}} \dot{\hat{\theta}}_{j}\right)
\end{aligned}
$$

where

$$
\begin{aligned}
\frac{\lambda b \tilde{\theta}_{n} \widehat{\theta}_{n}}{\gamma} \leq & \frac{\lambda b}{\gamma}\left(\frac{\theta_{n}^{2}}{2}-\frac{\tilde{\theta}_{n}^{2}}{2}\right) \leq \frac{\lambda b}{2 \gamma} \theta_{n}^{2}, \beta_{n}=b k_{n 1}\left(\frac{b Q_{n}^{2}}{\gamma}\right)^{(1+\alpha) / 2} \\
& +b k_{n 2}\left(\frac{b Q_{n}^{2}}{\gamma}\right)^{(1+\beta) / 2}, \\
\sigma_{n}= & \frac{\eta_{n}^{2}}{2}+\frac{\varepsilon_{n}^{2}}{4 b k_{n 3}}, \\
C_{n}= & \sigma_{n}+\beta_{n}+\frac{\lambda b \theta_{n}^{2}}{2 \gamma} .
\end{aligned}
$$

According to (21) and (22) and Remark 4, we consider only $\left|z_{n}\right| \geq \varepsilon_{n 0}$. Then, (73) can be written as

$$
\begin{aligned}
\dot{V}_{n} \leq & -b 2^{(1+\alpha) / 2} \sum_{j=1}^{n} k_{j 1}\left(\frac{1}{2} z_{j}^{2}+\frac{b \widetilde{\theta}_{j}^{2}}{2 \gamma}\right)^{(1+\alpha) / 2} \\
& -2 b \sum_{j=1}^{n} k_{j 2}\left(\frac{1}{2} z_{j}^{2}+\frac{b \widetilde{\theta}_{j}^{2}}{2 \gamma}\right)^{(1+\beta) / 2}+\sum_{j=1}^{n} C_{j} \\
& +z_{n}\left(M_{n-1}\left(Z_{n}\right)-\sum_{j=1}^{n-1} \frac{\partial \alpha_{k-1} \dot{\hat{\theta}}_{j}}{\partial \hat{\theta}_{j}}\right) .
\end{aligned}
$$

The treatment of $M_{n-1}\left(Z_{n}\right)$ is similar to that of (62) and (63), with $M_{n-1}\left(Z_{n}\right)$ being defined as

$$
\begin{aligned}
M_{n-1}\left(Z_{n}\right)= & -\sum_{j=1}^{n-1} \frac{z_{n}\left(\partial \alpha_{n-1} / \partial \hat{\theta}_{j}\right)^{2}\left(\left(\gamma / 2 \eta_{j}^{2}\right) s^{2} z_{j}^{2}\right)^{2}}{\sqrt{z_{n}^{2}\left(\partial \alpha_{n-1} / \partial \widehat{\theta}_{j}\right)^{2}\left(\left(\gamma / 2 \eta_{j}^{2}\right) s^{2} z_{j}^{2}\right)^{2}+\varsigma_{n, j}^{2}}} \\
& -\lambda \sum_{j=1}^{n-1} \frac{\partial \alpha_{n-1}}{\partial \widehat{\theta}_{j}} \widehat{\theta}_{j},
\end{aligned}
$$


with the result that

$$
z_{n}\left(M_{n-1}\left(Z_{n}\right)-\sum_{j=1}^{n-1} \frac{\partial \alpha_{n-1}}{\partial \widehat{\hat{\theta}}_{j}}\right) \leq 0
$$

Substituting (77) into (75) yields

$$
\dot{V}_{n} \leq-b 2^{(1+\alpha) / 2} \sum_{j=1}^{n} k_{j 1}\left(\frac{1}{2} z_{j}^{2}+\frac{b \widetilde{\theta}_{j}^{2}}{2 \gamma}\right)^{(1+\alpha) / 2}-2 b \sum_{j=1}^{n} k_{j 2}\left(\frac{1}{2} z_{j}^{2}+\frac{b \widetilde{\theta}_{j}^{2}}{2 \gamma}\right)^{(1+\beta) / 2}+\sum_{j=1}^{n} C_{j}
$$

where $\sum_{j=1}^{n} C_{j}=\tau$.

Making $\phi_{1}=\min 2^{(1+\alpha) / 2}\left(b k_{11}, b k_{21}, \ldots, b k_{n 1}\right) \quad$ and $\phi_{2}=\min 2\left(b k_{12}, b k_{22}, \ldots, b k_{n 2}\right)$, in accordance with Lemma 3 , yields

$$
\begin{aligned}
-b 2^{(1+\alpha) / 2} \sum_{j=1}^{n} k_{j 1}\left(\frac{1}{2} z_{j}^{2}+\frac{b \widetilde{\theta}_{j}^{2}}{2 \gamma}\right)^{(1+\alpha) / 2} \leq-\phi_{1}\left(\sum_{j=1}^{n}\left(\frac{1}{2} z_{j}^{2}+\frac{b \widetilde{\theta}_{j}^{2}}{2 \lambda}\right)\right)^{(1+\alpha) / 2}, \\
-2 b \sum_{j=1}^{n} k_{j 2}\left(\frac{1}{2} z_{j}^{2}+\frac{b \widetilde{\theta}_{j}^{2}}{2 \gamma}\right)^{(1+\beta) / 2} \leq-n^{(1-\beta) / 2} \phi_{2}\left(\sum_{j=1}^{n}\left(\frac{1}{2} z_{j}^{2}+\frac{b \widetilde{\theta}_{j}^{2}}{2 \gamma}\right)\right)^{(1+\beta) / 2}
\end{aligned}
$$

and then substituting (79) and (81) into (78) yields

$$
\dot{V}_{n} \leq-\phi_{1} V_{n}^{(1+\alpha) / 2}-n^{(1-\beta) / 2} \phi_{2} V_{n}^{(1+\beta) / 2}+\tau .
$$

Up to this point, the design of the controller is finished.

\section{Stability Analysis}

Theorem 1. If system (1) satisfies Assumptions 1-3 and uses the virtual controllers (27), (42), and (56), the actual controller (70), and the adaptive laws (32), (44), (58), and (72), all signals in the closed-loop system are semiglobally uniform and ultimately bounded, and the upper limit of the fixed convergence time is irrelevant to the initial state.

In accordance with Lemma 2, design proper parameters $k_{j 1}>0, k_{j 2}>0, k_{j 3}>0$, and $C_{j}>0, j=1, \ldots, n$, to make (81) satisfy the following situation.

Case 1. If $V_{n}>\left(\tau /\left((1-\varpi) \phi_{1}\right)\right)^{2 /(1+\alpha)}, \varpi \in(0,1)$, then $(81)$ can be written as

$$
\dot{V}_{n} \leq-\oplus \phi_{1} V_{n}^{(1+\alpha) / 2}-n^{(1-\beta) / 2} \phi_{2} V_{n}^{(1+\beta) / 2},
$$

and in that manner, the solution of system (1) converges on the compact set

$$
x \in\left\{V_{n}(x) \leq\left(\frac{\tau}{(1-\omega) \phi_{1}}\right)^{2 /(1+\alpha)}\right\}
$$

The fixed convergence time is

$$
T \leq T_{\max }:=\frac{2}{\omega \phi_{1}(1-\alpha)}+\frac{2 n^{(\beta-1) / 2}}{\phi_{2}(\beta-1)} .
$$

Case 2. If $V_{n} \geq\left(\tau n^{(\beta-1) / 2} /\left((1-\Phi) \phi_{2}\right)\right)^{2 /(1+\beta)}$, then (81) can be written as

$$
\dot{V}_{n} \leq-\phi_{1} V_{n}^{(1+\alpha) / 2}-\varpi n^{(1-\beta) / 2} \phi_{2} V_{n}^{(1+\beta) / 2},
$$

and in that manner, the solution of system (1) converges on the compact set

$$
x \in\left\{V_{n}(x) \leq\left(\frac{\tau n^{(\beta-1) / 2}}{(1-\varpi) \phi_{2}}\right)^{2 /(1+\beta)}\right\}
$$

The fixed convergence time is

$$
T \leq T_{\max }:=\frac{2}{\phi_{1}(1-\alpha)}+\frac{2 n^{(\beta-1) / 2}}{\omega \phi_{2}(\beta-1)} .
$$

When cases 1 and 2 are combined, the solution of system (1) converges on

$$
x \in\left\{V_{n}(x) \leq \min \left\{\left(\frac{\tau}{(1-\omega) \phi_{1}}\right)^{2 /(1+\alpha)},\left(\frac{\tau n^{(\beta-1) / 2}}{(1-\varpi) \phi_{2}}\right)^{2 /(1+\beta)}\right\}\right\} .
$$




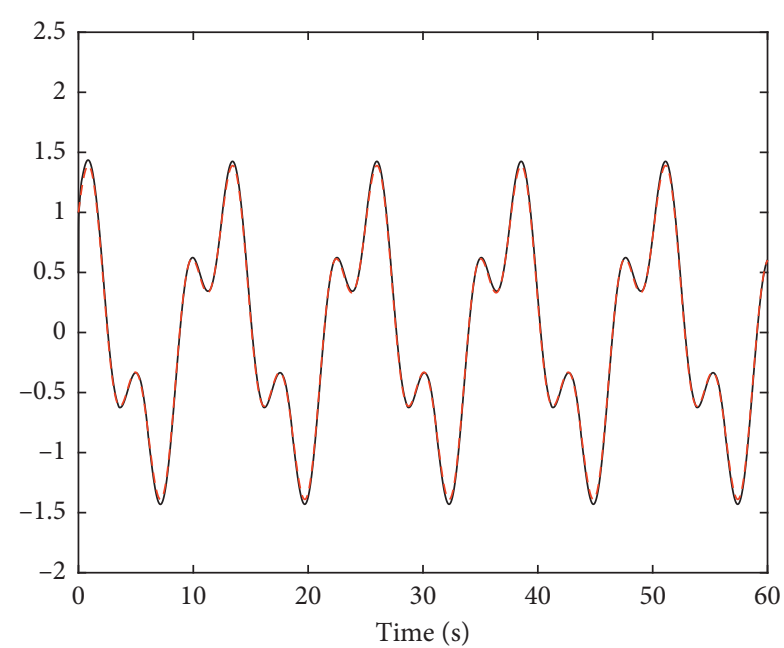

$-x_{1}$

$---y_{d}$

Figure 1: System output $y$ and reference signal $y_{d}$.

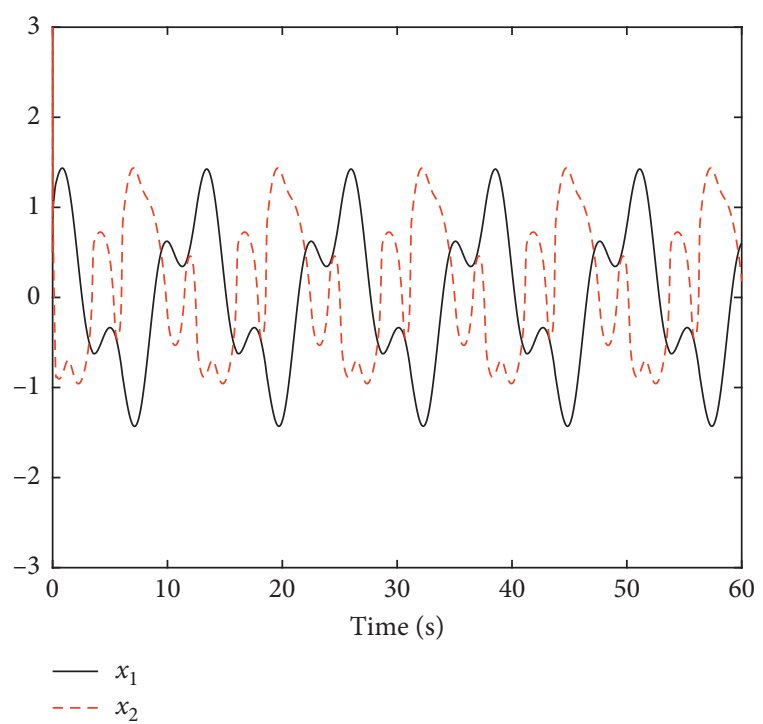

Figure 2: State variables $x_{1}$ and $x_{2}$.

The fixed convergence time is

$$
T_{s} \leq T_{\max }:=\frac{2}{\omega \phi_{1}(1-\alpha)}+\frac{2 n^{(\beta-1) / 2}}{\omega \phi_{2}(\beta-1)} .
$$

It can be seen from (82) and (85) that $V_{n}$ is bounded, with the result that $z_{j}$ and $\widetilde{\theta}_{j}$ are bounded. As $\widehat{\theta}_{j}=\theta_{j}-\widetilde{\theta}_{j}, \widehat{\theta}_{j}$ is also bounded, where $j=1, \ldots, n$. Because $z_{1}=x_{1}-y_{d}$ and $z_{1}$ and $y_{d}$ are bounded, $x_{1}$ is bounded. Because $\alpha_{1}$ is a function of $z_{1}, y_{d}, \dot{y}_{d}$, and $\widehat{\theta}_{1}, \alpha_{1}$ is bounded. Because $z_{2}=x_{2}-\alpha_{1}, \quad x_{2}$ is bounded. Similarly, $\alpha_{j-1}$ and $x_{j}$, $j=1, \ldots, n$, are bounded. Therefore, all signals in system (1) are bounded.

Remark 6. The fixed-time control algorithm in this paper is different from previous control algorithms. The principle differences are as follows:

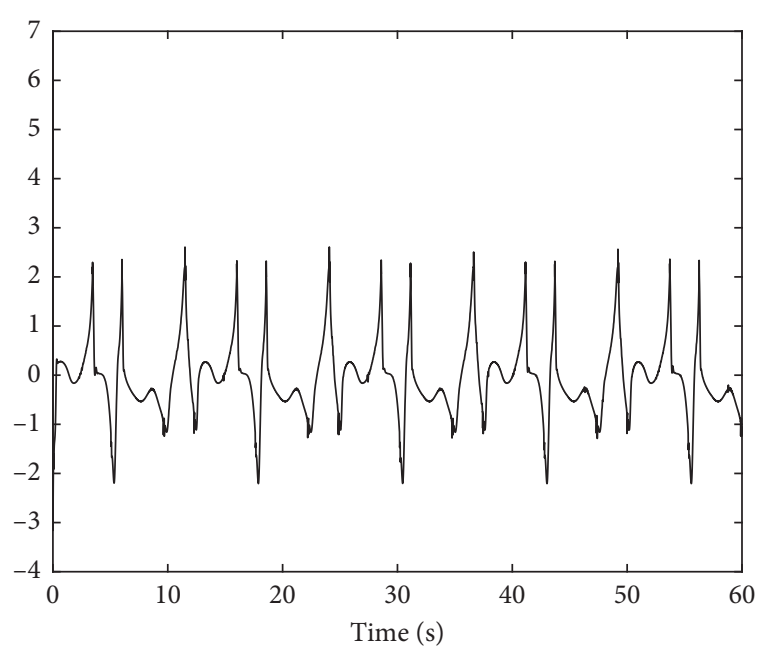

Figure 3: Actual control $u$.

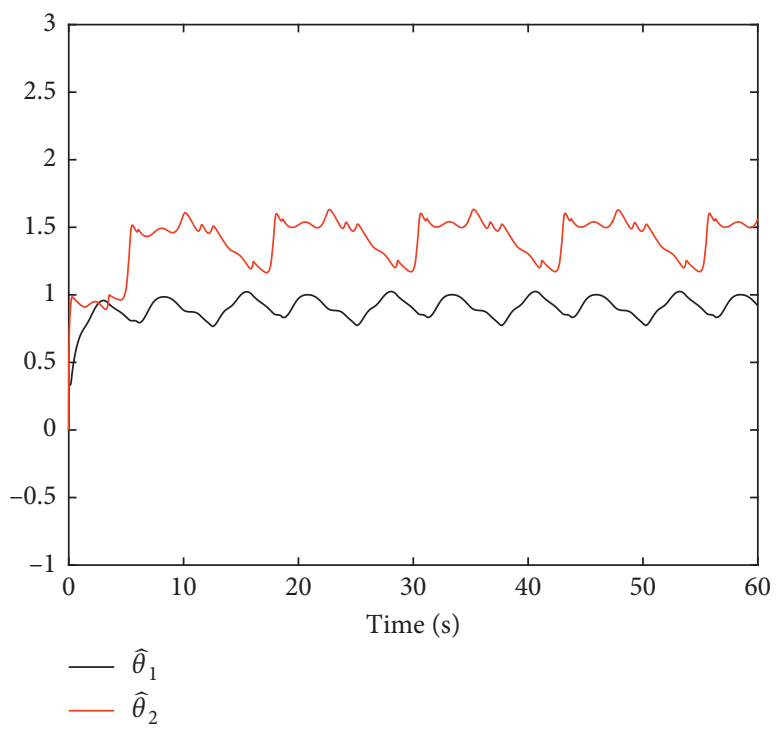

FIgure 4: Adaptive parameters $\widehat{\theta}_{1}$ and $\widehat{\theta}_{2}$.

(1) The fixed-time control algorithm proposed in [35-40] does not solve the problem of the nonaffine structure of the control input $u(t)$. The fixed-time control algorithm proposed here solves that problem.

(2) Some systems' $f_{i}(\cdot)$ structure is complex, which interferes with direct usage of $f_{i}(\cdot)$ for designing controllers. An RBF neural network is used here to approximate unknown functions $f_{i}(\cdot)$, thereby obviating the need to know the structure of $f_{i}\left(\bar{x}_{i}, x_{i+1}\right)$ and avoiding the difficult design problem of controllers derived from complex system structures.

\section{Simulation Results}

In this section, two samples are studied to verify the effectiveness of the controller designed in the paragraphs above. 


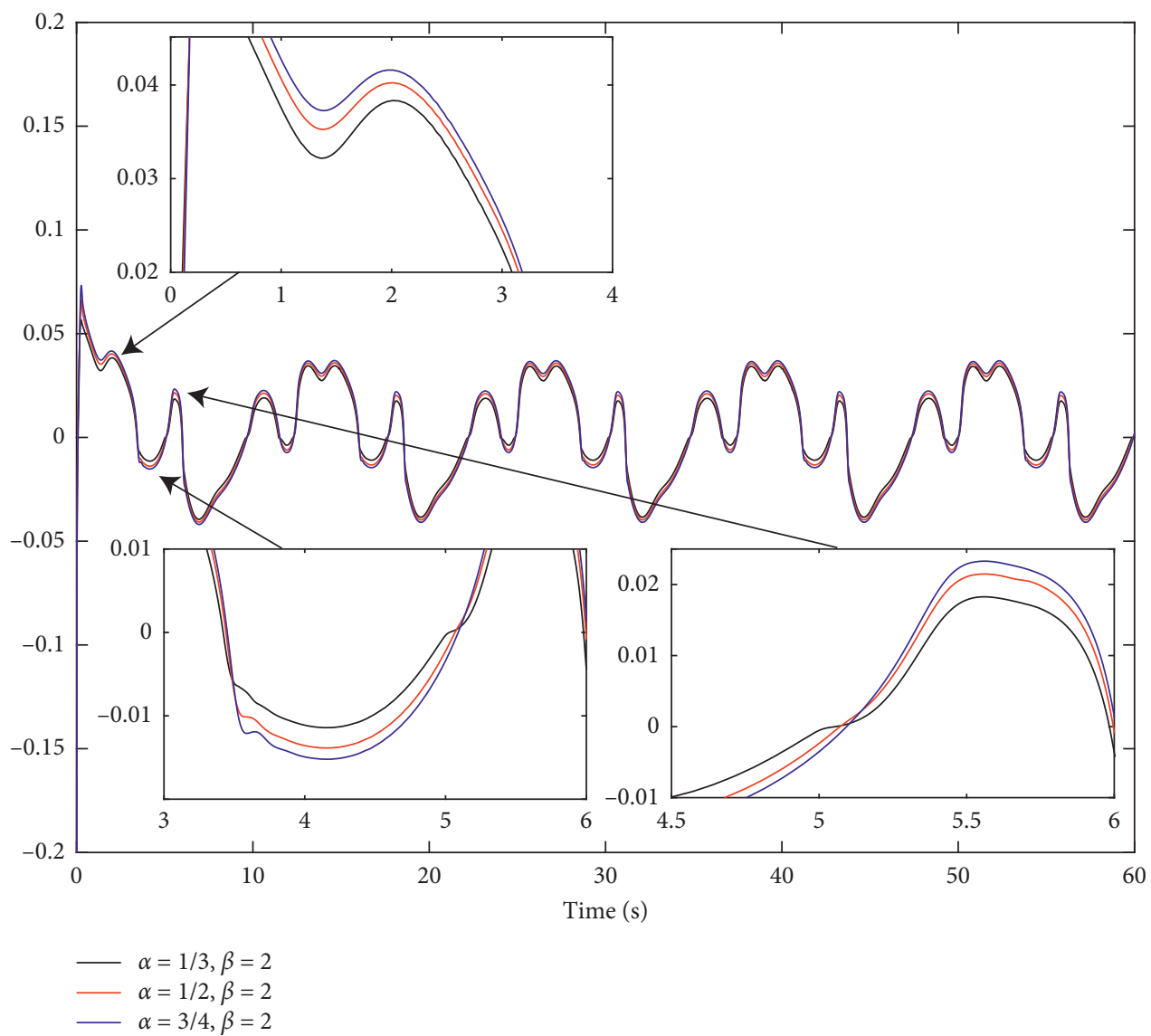

FiguRe 5: Tracking error $y-y_{d}$.

5.1. Mathematical Example. Consider the following nonlinear pure-feedback system:

$$
\left\{\begin{array}{l}
\dot{x}_{1}=\frac{1-e^{-x_{1}}}{1+e^{-x_{2}}}+x_{2}^{3}+x_{2} e^{-1-x_{1}^{2}}+d_{1}\left(\bar{x}_{1}, t\right), \\
\dot{x}_{2}=x_{1}^{2} x_{2}+\left(2 u+u^{3}\right)\left(x_{1}^{2}+x_{2}^{2}\right)+d_{2}\left(\bar{x}_{2}, t\right), \\
y=x_{1}
\end{array}\right.
$$

where $x_{1}$ and $x_{2}$ are the system state variables, $u$ is the system control input, $y$ is the system output, $d_{1}\left(\bar{x}_{1}, t\right)=$ $0.7 x_{1}^{2} \cos (1.5 t)$ and $d_{2}\left(\bar{x}_{2}, t\right)=0.5\left(x_{1}^{2}+x_{2}^{2}\right) \sin ^{3}(t) 0.5$ are the external disturbance terms, and $y_{d}=0.5 \sin (1.5 t)$ $+\cos (0.5 t)$ is the reference signal. It can be seen from system (90) that the state variables and the control input $(2 u+$ $\left.u^{3}\right)\left(x_{1}^{2}+x_{2}^{2}\right)$ have the nonaffine structures. The simulation study aims to design a fixed-time controller based on system (90) that ensures that the output signal $y$ can track the reference signal $y_{d}$.

For system (90), the fixed-time controller is defined as follows:

$$
\begin{aligned}
& \alpha_{1}=-k_{11} S_{z_{1}, 1}-k_{12} S_{z_{1}, 2}-\frac{\hat{\theta}_{1}}{2 \eta_{1}^{2}} S_{1}^{T}\left(Z_{1}\right) S_{1}\left(Z_{1}\right) z_{1}-k_{13} z_{1}+x_{10}, \\
& u=-k_{21} S_{z_{2}, 1}-k_{22} S_{z_{2}, 2}-\frac{\widehat{\theta}_{2}}{2 \eta_{2}^{2}} S_{2}^{T}\left(Z_{2}\right) S_{2}\left(Z_{2}\right) z_{2}-k_{23} z_{2}+x_{20}, \\
& \dot{\hat{\theta}}_{1}=\frac{\gamma}{2 \eta_{1}^{2}} S_{1}^{T}\left(Z_{1}\right) S_{1}\left(Z_{1}\right) z_{1}^{2}-\lambda \widehat{\theta}_{1}, \\
& \dot{\hat{\theta}}_{2}=\frac{\gamma}{2 \eta_{2}^{2}} S_{2}^{T}\left(Z_{2}\right) S_{2}\left(Z_{1}\right) z_{2}^{2}-\lambda \widehat{\theta}_{2},
\end{aligned}
$$

where $a_{1}=5 / 4, \quad a_{2}=-(1 / 4), \quad z_{1}=x_{1}-y_{d}, \quad z_{2}=x_{2}-\alpha_{1}$, $Z_{1}=\left[x_{1}, \widehat{\theta}_{1}, y_{d}, \dot{y}_{d}\right]$, and $Z_{2}=\left[x_{1}, x_{2}, \widehat{\theta}_{1}, \dot{y}_{d}, \ddot{y}_{d}\right]$.

Choose initial conditions as $\left[x_{1}(0), x_{2}(0)\right]^{T}=[0.5,0.5]^{T}$ and $\left[\widehat{\theta}_{1}(0), \widehat{\theta}_{2}(0)\right]^{T}=[0,0]^{T}$. The design parameters are chosen as follows: $\alpha=1 / 3, \beta=2, k_{11}=0.2, k_{12}=0.2$, $k_{13}=1, \quad k_{21}=0.2, \quad k_{22}=0.2, \quad k_{23}=1, \quad \gamma=5, \quad \eta_{1}=0.25$, $\eta_{2}=0.25, \varepsilon_{10}=0.001, \varepsilon_{20}=0.001, \lambda=0.1, x_{10}=0.2$, and $x_{20}=0.5$. We set the width of the RBF neural network as four. $W_{1} S_{1}\left(Z_{1}\right)$ and $W_{2} S_{2}\left(Z_{2}\right)$ contain seven and five nodes, respectively. The center of the Gaussian function is set as 


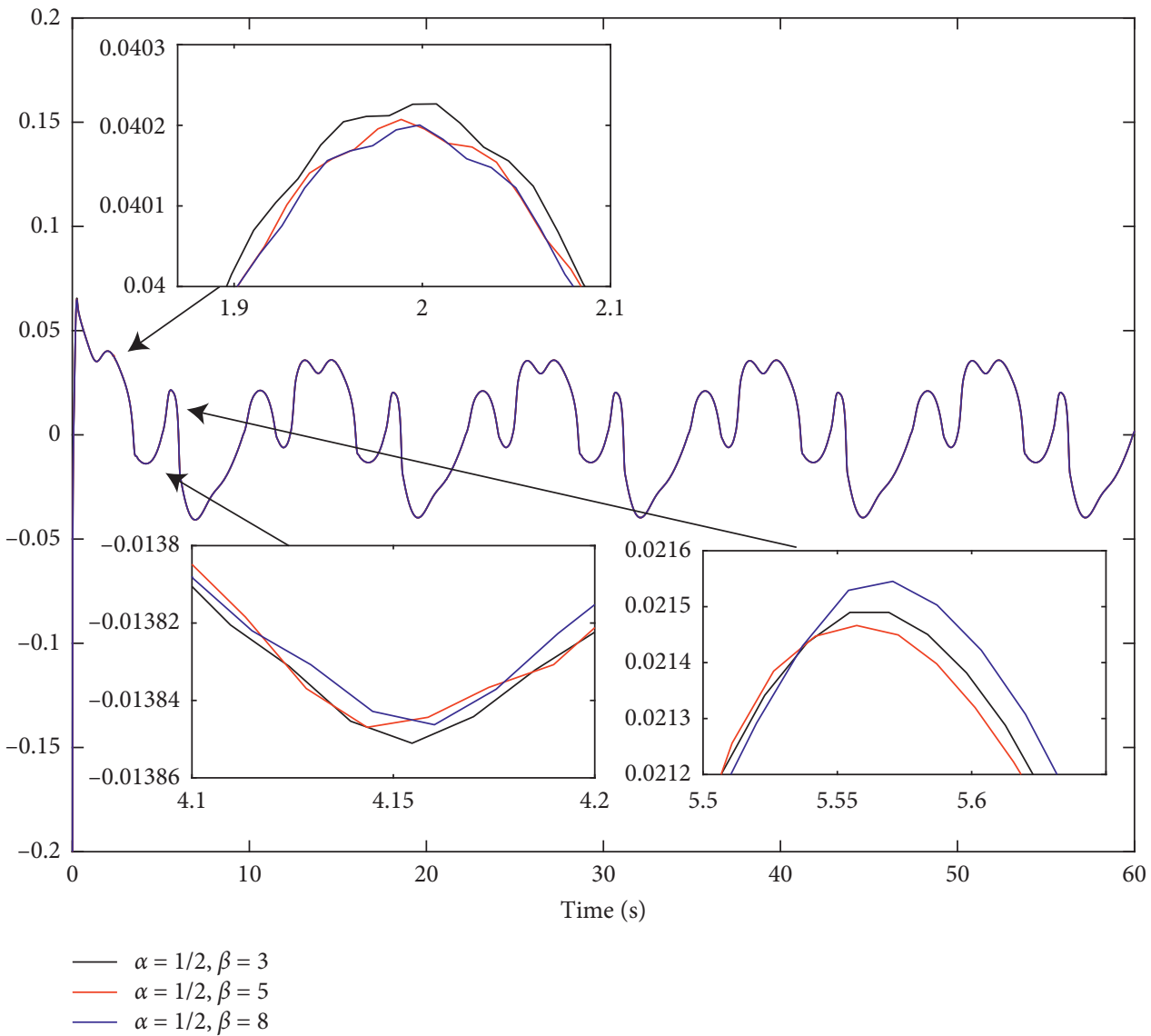

FIgURE 6: Tracking error $y-y_{d}$.

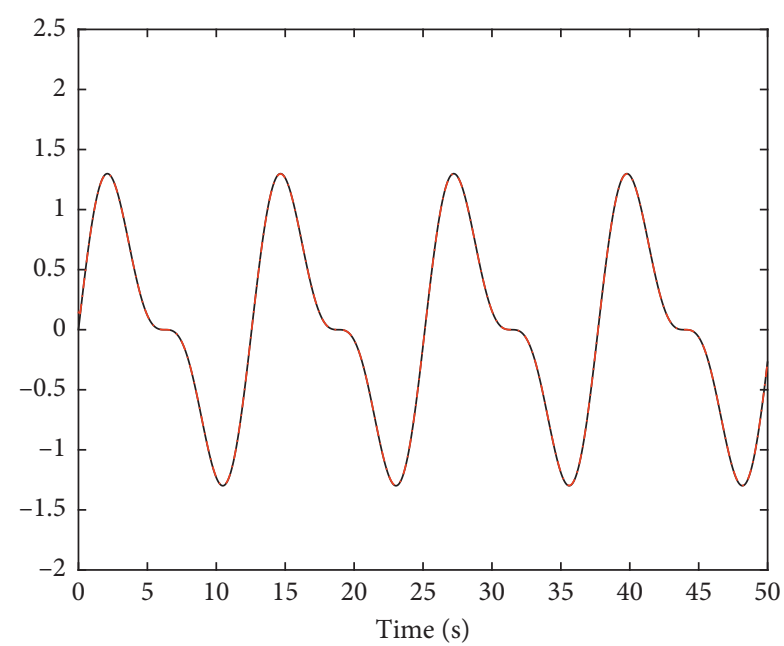

$-y_{d}$

FIGURE 7: System output $y$ and reference signal $y_{d}$.

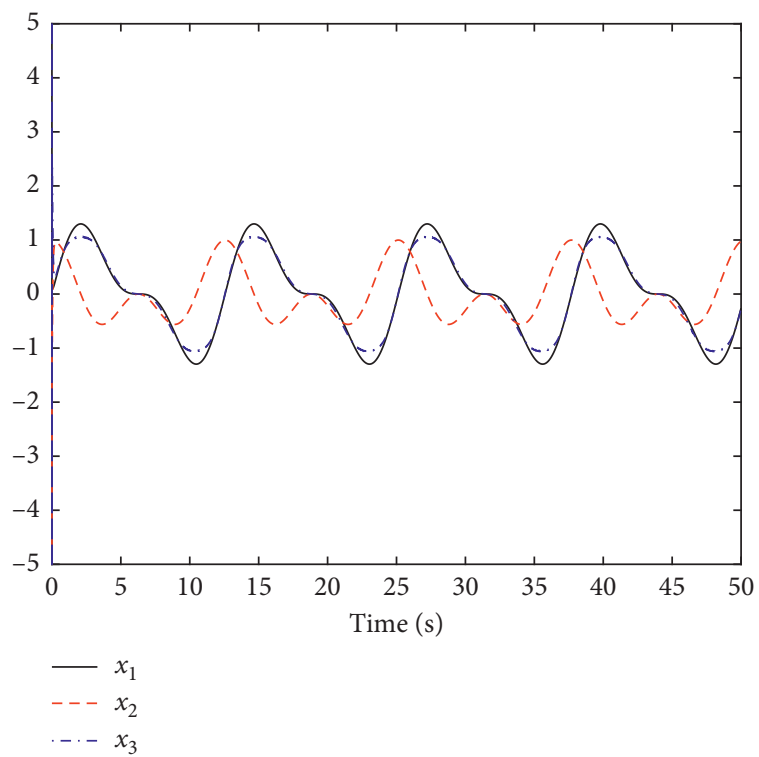

Figure 8: State variables $x_{1}, x_{2}$, and $x_{3}$. 


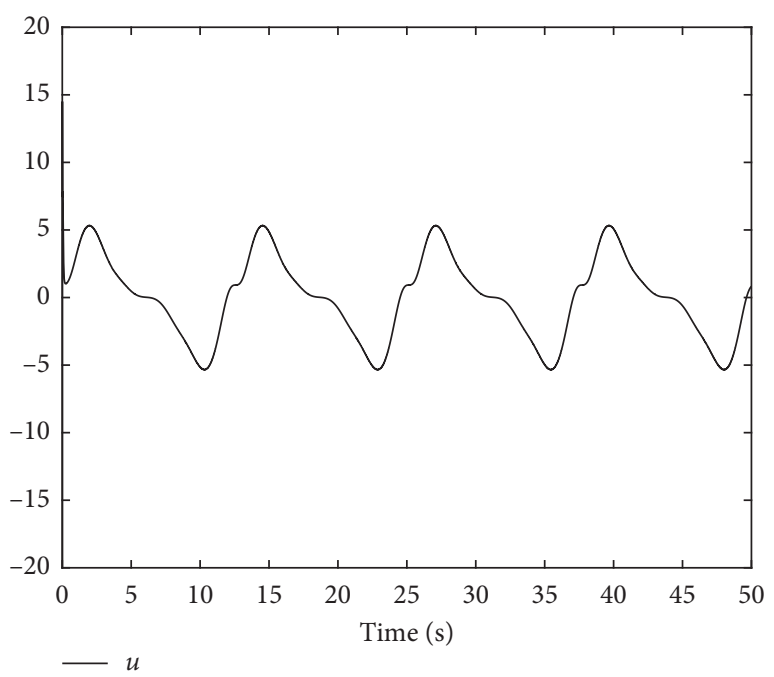

Figure 9: Actual control $u$.

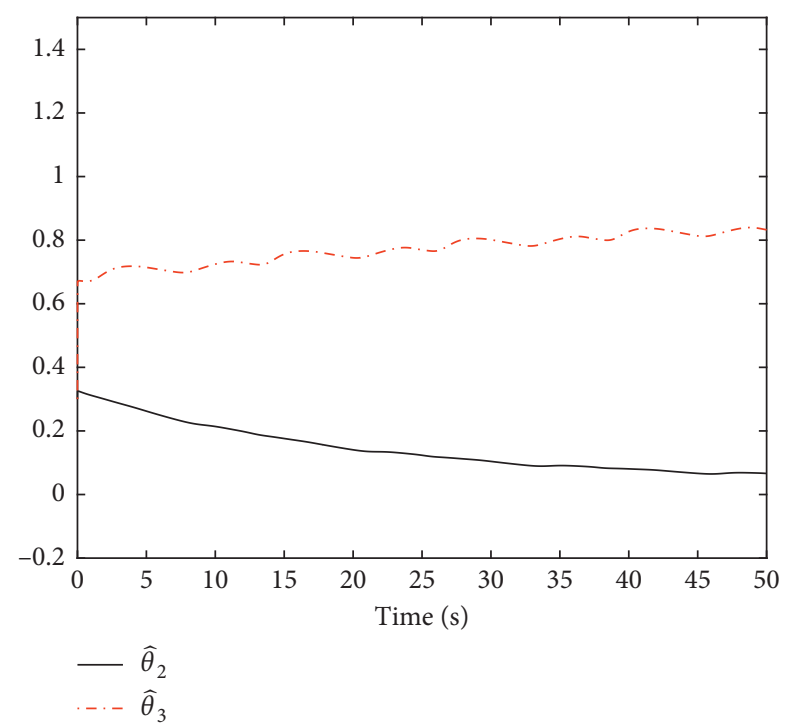

Figure 10: Adaptive parameters $\widehat{\theta}_{2}$ and $\widehat{\theta}_{3}$.

$$
\begin{aligned}
\xi_{1} & =\left[\begin{array}{rrrrrrr}
-2 & -1.5 & -1 & 0 & 1 & 1.5 & 2 \\
-2 & -1.5 & -1 & 0 & 1 & 1.5 & 2 \\
-2 & -1.5 & -1 & 0 & 1 & 1.5 & 2 \\
-2 & -1.5 & -1 & 0 & 1 & 1.5 & 2
\end{array}\right], \\
\xi_{2} & =\left[\begin{array}{lllll}
-2 & -1 & 0 & 1 & 2 \\
-2 & -1 & 0 & 1 & 2 \\
-2 & -1 & 0 & 1 & 2 \\
-3 & -2 & 0 & 2 & 3 \\
-3 & -2 & 0 & 2 & 3 \\
-3 & -2 & 0 & 2 & 3 \\
-3 & -2 & 0 & 2 & 3
\end{array}\right] .
\end{aligned}
$$

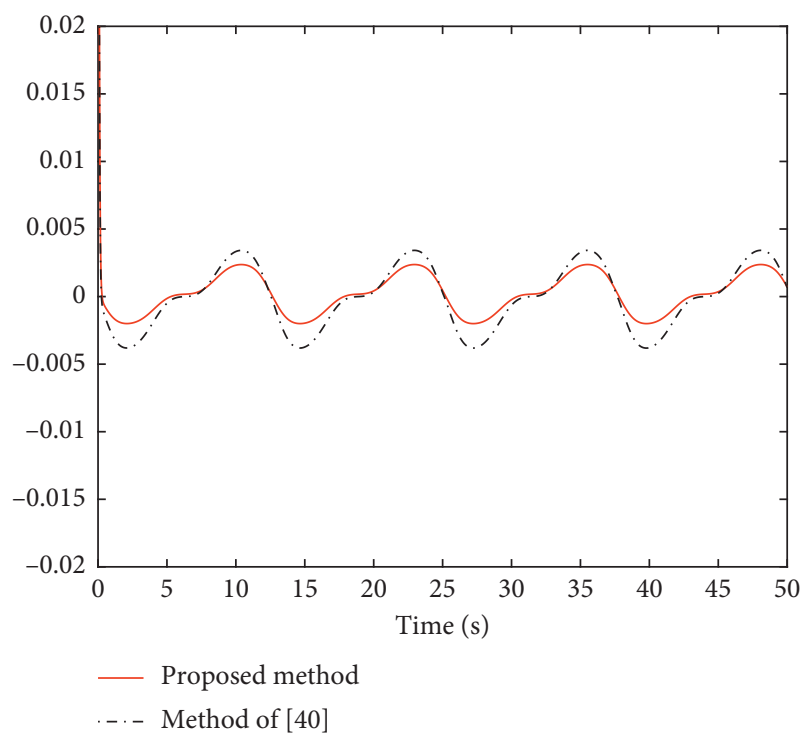

Figure 11: Tracking error $y-y_{d}$.

Figure 1 shows the system output $y$ and the reference signal $y_{d}$. It can be seen that $y$ can track reference signals $y_{d}$ effectively. Figures $2-4$ show the state variables $x_{1}$ and $x_{2}$, the actual controller $u$, and the adaptive parameters $\widehat{\theta}_{1}$ and $\widehat{\theta}_{2}$.

For a further simulation study, we selected two sets of different data to verify the tracking performance of system (90). It can be seen from Figure 5 that the error between the system output $y$ and the reference signal $y_{d}$ increases with an increase in the $\alpha$ value. It can be seen from Figure 6 that the error between the system output $y$ and the reference signal $y_{d}$ does not change significantly with an increase in the $\beta$ value.

Remark 7. In [40-43], because the controller has the power function $z^{2 \alpha-1}, \alpha$ can select only specific values; otherwise, the power function $z^{2 \alpha-1}$ is unsolvable at the origin and in the negative range. In this paper, $\alpha$ can be any number between zero and one.

It can be seen from Figures 1-6 that the state variables $x_{1}$ and $x_{2}$, the actual controller $u$, and the adaptive parameters $\widehat{\theta}_{1}$ and $\widehat{\theta}_{2}$ are bounded, with the consequence that all signals in the closed-loop system (90) are bounded.

5.2. Physical Example. Consider the following electromechanical system [41]:

$$
\left\{\begin{array}{l}
\dot{x}_{1}=x_{2}, \\
\dot{x}_{2}=e_{21} x_{2} x_{3}^{2}+e_{22} \sin x_{1}+e_{23} x_{2}-e_{23} x_{2}^{2} x_{3}^{3}, \\
\dot{x}_{3}=e_{31} u+e_{32} x_{2}+e_{33} x_{3}^{2}-e_{33} x_{2}^{2} \sin x_{3}, \\
y=x_{1},
\end{array}\right.
$$

where $e_{21}=1 / M, \quad e_{22}=-N / M, \quad e_{23}=-B / M, \quad e_{31}=1 / L$, $e_{32}=-K_{B} / L$, and $e_{33}=-R / L$ are system parameters. The 


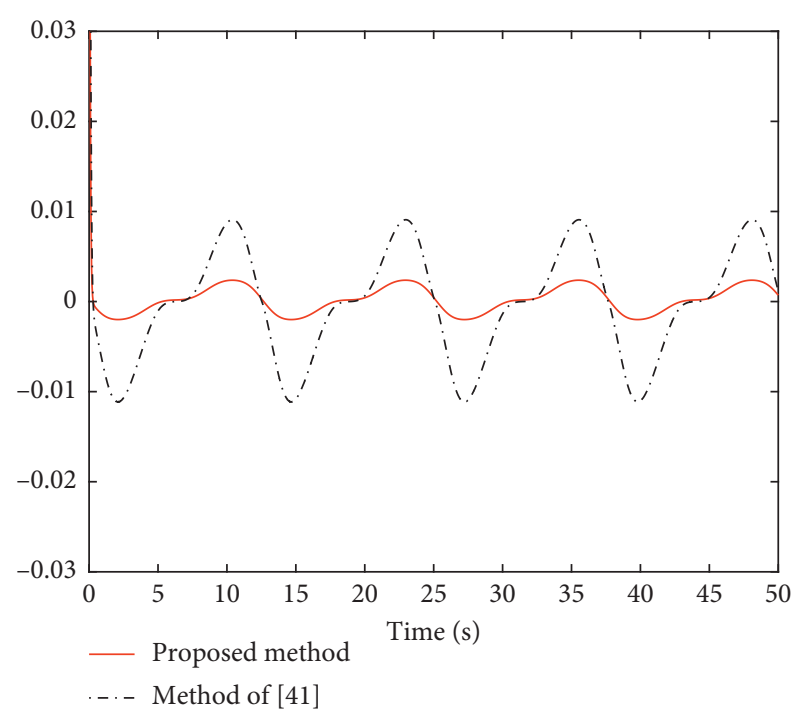

FIgURE 12: Tracking error $y-y_{d}$.

descriptions of $M, N, B, L, K_{B}$, and $R$ can be found in [53]. $y_{d}=\sin (0.5 t)+0.5 \sin (t)$ is the reference signal. The system parameters are chosen as follows: $M=0.0642, N=1.1408$, $B=0.0181, L=0.025, K_{B}=0.9$, and $R=5.0$. The simulation study aims to design a fixed-time controller based on system (93) to enable the output signal $y$ to track the reference signal $y_{d}$.

For system (93), the fixed-time controller is defined as follows:

$$
\begin{aligned}
& \alpha_{1}=-k_{11} S_{z_{1}, 1}-k_{12} S_{z_{1}, 2}+\dot{y}_{d}, \\
& \alpha_{2}=-k_{21} S_{z_{2}, 1}-k_{22} S_{z_{2}, 2}-\frac{\hat{\theta}_{2}}{2 \eta_{2}^{2}} S_{2}^{T}\left(Z_{2}\right) S_{2}\left(Z_{2}\right) z_{2}-k_{23} z_{2}+x_{20}, \\
& u=-k_{31} S_{z_{3}, 1}-k_{32} S_{z_{3}, 2}-\frac{\hat{\theta}_{3}}{2 \eta_{3}^{2}} S_{3}^{T}\left(Z_{3}\right) S_{3}\left(Z_{3}\right) z_{3}-k_{33} z_{3}+x_{30}, \\
& \dot{\hat{\theta}}_{2}=\frac{\gamma}{2 \eta_{2}^{2}} S_{2}^{T}\left(Z_{2}\right) S_{2}\left(Z_{1}\right) z_{2}^{2}-\lambda \widehat{\theta}_{2}, \\
& \dot{\hat{\theta}}_{3}=\frac{\gamma}{2 \eta_{3}^{2}} S_{3}^{T}\left(Z_{3}\right) S_{3}\left(Z_{3}\right) z_{3}^{2}-\lambda \widehat{\theta}_{3},
\end{aligned}
$$

where $a_{1}=45 / 32, a_{2}=-(18 / 32), a_{3}=5 / 32$, and $z_{i}=x_{i}-$ $\alpha_{i-1}$ with $i=1,2,3$.

To verify the superiority of our designed controller, the tracking performance of this controller is compared with those of a fixed-time controller [40] and a finite-time controller [41]. The same design parameters as [40] are used, as follows: $k_{11}=10, k_{12}=10, k_{21}=10, k_{22}=10, k_{31}=10$, $k_{32}=10, \quad k_{23}=10, \quad k_{33}=10, \quad \alpha=97 / 101, \quad \beta=107 / 100$, $x_{10}=0.1, x_{20}=0.1, x_{30}=0.1, \varepsilon_{10}=10^{-5}, \varepsilon_{20}=10^{-5}$, and $\varepsilon_{30}=10^{-5}$. The initial conditions are selected as $\left[x_{1}(0), x_{2}(0), x_{3}(0)\right]^{T}=[0.15,0.15,0.15]^{T} \quad$ and $\left[\widehat{\theta}_{2}(0), \widehat{\theta}_{3}(0)\right]=[0.3,0.3]^{T}$. The design parameters of the neural network are the same as [41].

Figure 7 shows the system output $y$ and the reference signal $y_{d}$. As can be seen from Figure 7, the system output $y$ can track reference signals $y_{d}$ effectively. Figures 8-10 show the state variables, the actual controller, and the adaptive parameters, respectively. Figure 11 shows the tracking error comparison between the fixed-time controller in this paper and the fixed-time controller in [40]. Figure 12 shows the tracking error comparison between the fixed-time controller in this paper and the finite-time controller in [41]. It can be seen from the simulation diagram that the performance of the fixed-time controller proposed in this paper is superior.

It can be seen from Figures 7-12 that the state variables $x_{1}$ and $x_{2}$, the actual controller $u$, and the adaptive parameters $\hat{\theta}_{1}$ and $\widehat{\theta}_{2}$ are bounded, with the result that all signals in the closed-loop system (93) are bounded.

\section{Conclusion}

A novel virtual controller and a novel actual controller designed in this paper solve the singularity problem of the virtual controller and the actual controller at the origin and in the negative domain. The fixed-time controller designed is applied to nonlinear pure-feedback systems and solves the problem of nonaffine structure. The controller enables the system output to track the reference signal in a fixed time and also to make the tracking error converge to a region of the origin in a fixed time. The simulation results prove the efficiency of the controller designed in this paper. We intend to apply the approximation-based fixed-time adaptive tracking control for a class of uncertain nonlinear purefeedback systems in time-delay systems in the future.

\section{Data Availability}

No data were used to support this study.

\section{Conflicts of Interest}

The authors declare that they have no conflicts of interest.

\section{Acknowledgments}

This study was supported in part by the National Natural Science Foundation of China under grant no. 61663032 and by the Aviation Science Foundation of China under grant no. 2016 ZC56003.

\section{References}

[1] S. Labiod and T. M. Guerra, "Adaptive fuzzy control of a class of SISO nonaffine nonlinear systems," Fuzzy Sets and Systems, vol. 158, no. 10, pp. 1126-1137, 2007.

[2] Q. Kang, W. Wang, and Y. Liu, "Adaptive robust fuzzy control for a class of uncertain nonlinear systems in pure-feedback form," Automatica, vol. 38, no. 8, pp. 1365-1372, 2002.

[3] D. S. Broomhead and D. Lowe, "Multivariable functional interpolation and adaptive networks," Complex Systems, vol. 2, no. 3, pp. 321-355, 1988.

[4] A. R. Barron, "Universal approximation bounds for superpositions of a sigmoidal function," IEEE Transactions on Information Theory, vol. 39, no. 3, pp. 930-945, 1993.

[5] X. Liu and T. Chen, "A new result on the global convergence of Hopfield neural networks," IEEE Transactions on Circuits 
and Systems I Fundamental Theory and Applications, vol. 49, no. 10 , pp. 1514-1516, 2002.

[6] K. Hornic, "Multilayer feedforward networks are universal approximators," Neural Networks, vol. 2, no. 5, pp. 359-366, 1989.

[7] T. Poggio and F. Girosi, "Networks for approximation and learning," Proceedings of the IEEE, vol. 78, no. 9, pp. 1481-1497, 1990.

[8] I. Kanellakopoulos, P. V. Kokotovic, and A. S. Morse, "Systematic design of adaptive controllers for feedback linearizable systems," IEEE Transactions on Automatic Control, vol. 36, no. 11, pp. 1241-1253, 1991.

[9] M. Krstic, I. Kanellakopoulos, and P. V. Kokotovic, “Adaptive nonlinear control without overparametrization," Systems \& Control Letters, vol. 19, no. 3, pp. 177-185, 1992.

[10] C. Kwan and F. L. Lewis, "Robust backstepping control of nonlinear systems using neural networks," IEEE Transactions on Systems, Man, and Cybernetics-Part A: Systems and Humans, vol. 30, no. 6, pp. 753-766, 2000.

[11] R. M. Sanner and J.-J. E. Slotine, "Gaussian networks for direct adaptive control," IEEE Transactions on Neural Networks, vol. 3, no. 6, pp. 837-863, 1992.

[12] S. Seshagiri and H. K. Khalil, "Output feedback control of nonlinear systems using RBF neural networks," IEEE Transactions on Neural Networks, vol. 11, no. 1, pp. 69-79, 2000.

[13] S. N. Huang, K. K. Tan, and T. H. Lee, "Adaptive motion control using neural network approximations," Automatica, vol. 38, no. 2, pp. 227-233, 2002.

[14] S. S. Ge and C. Cong Wang, "Direct adaptive NN control of a class of nonlinear systems," IEEE Transactions on Neural Networks, vol. 13, no. 1, pp. 214-221, 2002.

[15] Y. Li, S. Qiang, X. Zhuang, and O. Kaynak, "Robust and adaptive backstepping control for nonlinear systems using RBF neural networks," IEEE Transactions on Neural Networks, vol. 15, no. 3, pp. 693-701, 2004.

[16] S. Huang, K. K. Tan, T. H. Lee, and A. S. Putra, "Adaptive control of mechanical systems using neural networks," IEEE Transactions on Systems, Man and Cybernetics, Part C (Applications and Reviews), vol. 37, no. 5, pp. 897-903, 2007.

[17] S. Wang and D. L. Yu, "Adaptive RBF network for parameter estimation and stable air-fuel ratio control," Neural Networks, vol. 21, no. 1, pp. 102-112, 2008.

[18] Q. Zhu, S. Fei, T. Zhang, and T. Li, "Adaptive RBF neuralnetworks control for a class of time-delay nonlinear systems," Neurocomputing, vol. 71, no. 16-18, pp. 3617-3624, 2008.

[19] T. Zhang and S. S. Ge, "Adaptive neural network tracking control of MIMO nonlinear systems with unknown dead zones and control directions," IEEE Transactions on Neural Networks, vol. 20, no. 3, pp. 483-497, 2009.

[20] S.-H. Yu and A. M. Annaswamy, "Adaptive control of nonlinear dynamic systems using $\theta$-adaptive neural networks," Automatica, vol. 33, no. 11, pp. 1975-1995, 1997.

[21] S.-H. Yu and A. M. Annaswamy, "Stable neural controllers for nonlinear dynamic systems," Automatica, vol. 34, no. 5, pp. 641-650, 1998.

[22] E. RANG, "Isochrone families for second-order systems," IEEE Transactions on Automatic Control, vol. 8, no. 1, pp. 64-65, 1963.

[23] S. P. Bhat and D. S. Bernstein, "Lyapunov analysis of finitetime differential equations," Proceedings of the American Control Conference IEEE, pp. 1831-1832, 1995.
[24] S. P. Bhat and D. S. Bernstein, "Finite-time stability of homogeneous systems," Proceedings of the American Control Conference, pp. 2513-2514, 1997.

[25] C. Hua, Y. Li, and X. Guan, "Finite/fixed-time stabilization for nonlinear interconnected systems with dead-zone input," IEEE Transactions on Automatic Control, vol. 62, no. 5, pp. 2554-2560, 2017.

[26] H. Du, S. Li, and C. Qian, "Finite-time attitude tracking control of spacecraft with application to attitude synchronization," IEEE Transactions on Automatic Control, vol. 56, no. 11, pp. 2711-2717, 2011.

[27] S. Wu, G. Radice, Y. Gao, and Z. Sun, "Quaternion-based finite time control for spacecraft attitude tracking," Acta Astronautica, vol. 69, no. 1-2, pp. 48-58, 2011.

[28] M. S. Song, Y. Guo, and H. X. Li, "Finite-time attitude tracking control for spacecraft with input saturation," Control and Decision, vol. 30, no. 11, pp. 2004-2008, 2015.

[29] C. Wang and Y. Wu, "Finite-time tracking control for strictfeedback nonlinear systems with full state constraints," International Journal of Control, vol. 92, no. 6, pp. 1426-1433, 2019.

[30] Q. Hui, W. M. Haddad, and S. P. Bhat, "Finite-time semistability and consensus for nonlinear dynamical networks," IEEE Transactions on Automatic Control, vol. 53, no. 8, pp. 1887-1900, 2008.

[31] A. Polyakov, "Nonlinear feedback design for fixed-time stabilization of linear control systems," IEEE Transactions on Automatic Control, vol. 57, no. 8, pp. 2106-2110, 2012.

[32] F. Gao, Y. Wu, and Z. Zhang, "Global fixed-time stabilization of switched nonlinear systems: a time-varying scaling transformation approach," IEEE Transactions on Circuits and Systems II: Express Briefs, vol. 66, no. 11, pp. 1890-1894, 2019.

[33] A. Polyakov, D. Efimov, and W. Perruquetti, "Finite-time and fixed-time stabilization: implicit Lyapunov function approach," Automatica, vol. 51, pp. 332-340, 2015.

[34] Z. Zhang and Y. Wu, "Fixed-time regulation control of uncertain nonholonomic systems and its applications," International Journal of Control, vol. 90, no. 7, pp. 1327-1344, 2017.

[35] H. Wang, W. Yu, G. Wen, and G. Chen, "Fixed-time consensus of nonlinear multi-agent systems with general directed topologies," IEEE Transactions on Circuits and Systems II: Express Briefs, vol. 66, no. 9, pp. 1587-1591, 2019.

[36] J. Ni, C. K. Ahn, L. Liu, and C. Liu, "Prescribed performance fixed-time recurrent neural network control for uncertain nonlinear systems," Neurocomputing, vol. 363, pp. 351-365, 2019.

[37] J. Ni, L. Liu, C. Liu, X. Hu, and S. Li, "Fast fixed-time nonsingular terminal sliding mode control and its application to chaos suppression in power system," IEEE Transactions on Circuits and Systems II: Express Briefs, vol. 64, no. 2, pp. 151-155, 2017.

[38] Y. Wu, Z. Wang, and Z. Huang, "Distributed fault detection for nonlinear multi-agent systems under fixed-time observer," Journal of the Franklin Institute, vol. 356, no. 13, pp. 75157532, 2019.

[39] J. Li, Y. Yang, C. Hua, and X. Guan, "Fixed-time backstepping control design for high-order strict-feedback non-linear systems via terminal sliding mode," IET Control Theory \& Applications, vol. 11, no. 8, pp. 1184-1193, 2017.

[40] D. Ba, Y.-X. Li, and S. Tong, "Fixed-time adaptive neural tracking control for a class of uncertain nonstrict nonlinear systems," Neurocomputing, vol. 363, pp. 273-280, 2019.

[41] Y. Sun, B. Chen, C. Lin, and H. Wang, "Finite-time adaptive control for a class of nonlinear systems with nonstrict 
feedback structure," IEEE Transactions on Cybernetics, vol. 48, no. 10, pp. 2774-2782, 2018.

[42] F. Wang, X. Zhang, B. Chen, C. Lin, X. Li, and J. Zhang, "Adaptive finite-time tracking control of switched nonlinear systems," Information Sciences, vol. 421, pp. 126-135, 2017.

[43] F. Wang, B. Chen, X. Liu, and C. Lin, "Finite-time adaptive fuzzy tracking control design for nonlinear systems," IEEE Transactions on Fuzzy Systems, vol. 26, no. 3, pp. 1207-1216, 2018.

[44] T. M. Apostol, Mathematical Analysis, Addison-Wesley, Reading, MA, USA, 1963.

[45] M. Wang, S. S. Ge, and K.-S. Hong, "Approximation-based adaptive tracking control of pure-feedback nonlinear systems with multiple unknown time-varying delays," Ieee Transactions on Neural Networks, vol. 21, no. 11, pp. 1804-1816, 2010.

[46] Z. Zuo, B. Tian, M. Defoort, and Z. Ding, "Fixed-time consensus tracking for multiagent systems with high-order integrator dynamics," IEEE Transactions on Automatic Control, vol. 63 , no. 2, pp. 563-570, 2018.

[47] G. H. Hardy, J. E. Littlewood, and G. Polya, Inequalities, Cambridge University Press, Cambridge, UK, 1952.

[48] C. Wang and Y. Lin, "Decentralized adaptive tracking control for a class of interconnected nonlinear time-varying systems," Automatica, vol. 54, pp. 16-24, 2015.

[49] H. Du, H. Shao, and P. Yao, "Adaptive neural network control for a class of low-triangular-structured nonlinear systems," IEEE Transactions on Neural Networks, vol. 17, no. 2, pp. 509-514, 2006.

[50] A. J. Kurdila, F. J. Narcowich, and J. D. Ward, "Persistency of excitation in identification using radial basis function approximants," SIAM Journal on Control and Optimization, vol. 33, no. 2, pp. 625-642, 1995.

[51] C. Wang, D. J. Hill, S. S. Ge, and G. Chen, "An ISS-modular approach for adaptive neural control of pure-feedback systems," Automatica, vol. 42, no. 5, pp. 723-731, 2006.

[52] J. Xu, "Adaptive fixed-time control for MIMO nonlinear systems with asymmetric output constraints using universal barrier functions," IEEE Transactions on Automatic Control, 2018.

[53] D. M. Dawson, J. J. Carroll, and M. Schneider, "Integrator backstepping control of a brush DC motor turning a robotic load," IEEE Transactions on Control Systems Technology, vol. 2, no. 3, pp. 233-244, 1994. 